\title{
Dense Motion Propagation from Sparse Samples
}

\author{
Rhodri L Smith ${ }^{1}$, Paul Dasari ${ }^{2}$, Clifford Lindsay ${ }^{2}$, \\ Michael King ${ }^{2}$ and Kevin Wells ${ }^{1}$ \\ ${ }^{1}$ Centre for Vision Speech and Signal Processing, Faculty of Engineering and Physical \\ Sciences, University of Surrey, Guildford, Surrey, GU2 7XH, UK. \\ ${ }^{2}$ Department of Radiology, Division of Nuclear Medicine, University of Massachusetts \\ Medical School, 55 Lake Ave North, Worcester, MA 01655
}

\begin{abstract}
There are many applications for which sparse, or partial sampling of dynamic image data can be used for articulating or estimating motion within the medical imaging area. In this new work, we propose a generalized framework for dense motion propagation from sparse samples which represents an example of transfer learning and manifold alignment, allowing the transfer of knowledge across imaging sources of different domains which exhibit different features. Many such examples exist in medical imaging, from mapping $2 \mathrm{D}$ ultrasound or fluoroscopy to $3 \mathrm{D}$ or $4 \mathrm{D}$ data or monitoring dynamic dose delivery from partial imaging data in radiotherapy. To illustrate this approach we animate, or articulate, a high resolution static MR image with $4 \mathrm{D}$ free breathing respiratory motion derived from low resolution sparse planar samples of motion. In this work we demonstrate that sparse sampling of dynamic MRI may be used as a viable approach to successfully build models of freebreathing respiratory motion by constrained articulation. Such models demonstrate high contrast, and high temporal and spatial resolution that have so far been hitherto unavailable with conventional imaging methods. The articulation is based on using a propagation model, in the eigen domain, to estimate complete $4 \mathrm{D}$ motion vector fields from sparsely sampled free-breathing dynamic MRI data. We demonstrate that this approach can provide equivalent motion vector fields compared to fully sampled $4 \mathrm{D}$ dynamic data, whilst preserving the corresponding high resolution / high contrast inherent in the original static volume. Validation is performed on three 4D MRI datasets using 8 extracted slices from a fast $4 \mathrm{D}$ acquisition ( $0.7 \mathrm{sec}$ per volume). The estimated deformation fields from sparse sampling are compared to the fully sampled equivalents, resulting in an rms error of the order of $2 \mathrm{~mm}$ across the entire image volume. We also present exemplar 4D high contrast, high resolution articulated volunteer datasets using this methodology. This approach facilitates greater freedom in the acquisition of free breathing respiratory motion sequences which may be used to inform motion modelling methods in a range of imaging modalities and demonstrates that sparse sampling of free breathing data may be used within a manifold alignment and transfer learning paradigm to estimate fully sampled motion. The method may also be applied to other examples of sparse sampling to produce dense motion propagation.
\end{abstract}

\section{Introduction}

Internal abdominal thoracic motion caused by respiration results in internal organ, and associated lesion motion, with a magnitude of $\approx 5-25 \mathrm{~mm}$, mainly in the superior- 
inferior and posterior-anterior directions (Dawood et al. 2006). Respiratory organ motion thus degrades the accuracy of diagnostic imaging ( $\mathrm{Xu}$ et al. 2011, Faranesh et al. 2013, Polycarpou et al. 2014), image guided interventional procedures (King et al. 2009) and radiotherapy treatment (Wilms et al. 2013). This results in artefacts in reconstructed images, inaccuracies in image guidance and internal radiotherapy target volumes. Respiratory motion during free breathing has also demonstrated itself to be a non stationary process (Keall et al. 2006). Correction methods which therefore group imaging data into one average respiratory cycle (Bai et al. 2009, Liu et al. 2011) neglect inherent inter-cycle variabilities in respiratory motion (Blackall et al. 2006, Biederer et al. 2017, Lagendijk 2007). Inter-cycle variabilities refer to differences between separate cycles and can be due to changes in breathing amplitude (inhaling/exhaling more or less air), or due to changes in breathing style (so called "belly breathing" versus "chest breathing"). These variabilities can compromise the quality of the resulting corrected images and is the motivation behind the design of recent motion models developed to account for this (Baumgartner et al. 2017, King et al. 2012). Respiratory motion models offer an attempt to alleviate the detriment in the aforementioned areas, by approximating the relationship between a surrogate signal and the motion of interest using a correspondence model. The surrogate data should be easily measurable and have a strong relationship with the motion of interest (McClelland et al. 2012). It has recently been demonstrated that multi-dimensional surrogate signals are better able to capture the complex volumetric nature of internal motion compared with simple one dimensional surrogate signals (e.g. abdominal pressure belts, spirometer-based methods etc) when utilizing correspondence models (Fayad et al. 2012, Wilms et al. 2013, Barnes et al. 2008). The development of models of respiration for motion correction in diagnostic imaging have often utilized dynamic 4D data, on which plausible respiratory motion patterns can be estimated or validated.

To date, dynamic 4D MRI has often been used, its attraction lying in its zero radiation burden, large field of view and high soft tissue contrast. Correction of positron emission tomography (PET) images, with their inherent long acquisition times is a particular application focus of this work and more pertinent with the advent of $\mathrm{PET} / \mathrm{MR}$ scanners which brings with it the potential to correct for motion artefacts during simultaneous PET/MR imaging. Real time imaging data has the advantage of revealing both inter and intra cycle variability. The desired MR imaging protocol should both capture data with sufficient temporal resolution $(<1 s)$ to adequately sample volumetric free breathing patterns whilst having sufficient image quality or fidelity to visualize the internal non-rigid abdominal-thoracic motion. Due to limitations in scanner technology clinically practical acquisitions of this nature prove troublesome (Cai et al. 2011). Compressed sensing approaches to signal acquisition in MR, for example Kustner et al. 2016, are promising areas of future work in this area however they have the associated complexity of bringing compressed sensing into routine clinical use in MR (Hollingsworth et al. 2015). Thus to date, such MR images are often found exhibiting blurring or ghosting artefacts in the acquired images or lack the excellent soft 
tissue contrast found with static imaging methods. However the MR data itself, can, nonetheless, be used as a surrogate measure of respiratory motion and coupled with a motion model. As highlighted (McClelland et al. 2012) even the use of MR imaging data in this manner is limited (King et al. 2012). Dynamic 2D scans however can be acquired in a shorter time frame with adequate image quality. Retrospective reconstruction of dynamic 3D volumes may then be achieved by stacking or re-binning 2D slices which are acquired multiple times in a slice-by-slice fashion (Dikaios et al., 2014, von Siebenthal et al., 2007). More complex methods of slice stacking have very recently been proposed (Baumgartner et al. 2014), as highlighted, however, re-binning data in such a manner removes the ability to model inter-cycle variability which may be particularly apparent in patients that breath irregularly due to pathology, anxiety etc. Moreover, healthy individuals have also been found to exhibit irregular breathing patterns (Tahavori et al. 2014). Furthermore an issue for lengthy PET/MR data acquisitions may also be the amount of MRI data acquired and available to monitor motion (Fayad et al. 2015). There is also an in-ability to acquire diagnostic quality MRI simultaneously as imaging data that are used to monitor respiratory motion. It has therefore been proposed that the ultimate motion correction protocol used for PET/MRI may involve a combination of 3D and 2D MRI acquisitions (Dikaios et al. 2014) coupled with the use of partial or sparse imaging data in order to estimate full 3D motion (Mcclelland et al. 2014).

Thus the motivation of this work is to introduce a generalizable framework to build an articulated virtual model or animation of, in this case, abdominal-thoracic motion whilst the patient is in continuous free breathing utilizing both 3D and sparse 2D image acquisitions. We provide the first demonstration that sparse sampling of, in this particular example, dynamic MRI may be used to successfully build models of whole body free-breathing respiratory motion whilst also delivering high contrast to noise ratio (CNR) and high spatial resolution and fine (sub second) temporal sampling. This may be used to inform fundamental understanding on the patterns of internal respiratory motion and also aid the development of motion correction and motion prediction image acquisition and correction methodology. With an a-priori eigenbasis of respiratory motion we demonstrate that only sparse sampling of motion is required to fully and accurately articulate the abdominal thoracic organs. Our method thus has four advantages: Firstly it removes the need for continuously acquiring fully sampled free breathing dynamic sequences which, with MRI, fundamentally exhibit low image contrast and low signal to noise ratio; secondly it allows the realization of high image quality $4 \mathrm{D}$ datasets which may be used as the basis for building generalizable models of respiratory motion. Thirdly the framework may also be developed to correct motion artefacts of individual subjects directly; finally we provide a mechanism for knowledge transfer between different domains allowing different sources of image motion with different features to be utilized in the development of respiratory motion modelling, thus rendering the approach generalizable across different combinations of imaging modalities and surrogate signals. This allows the application of a variety of different internal / external surrogate signals which may become available during PET/MR imaging (e.g. 
navigator and / or external monitoring) and the incorporation of these at different periods dependent upon their availability during image acquisition.

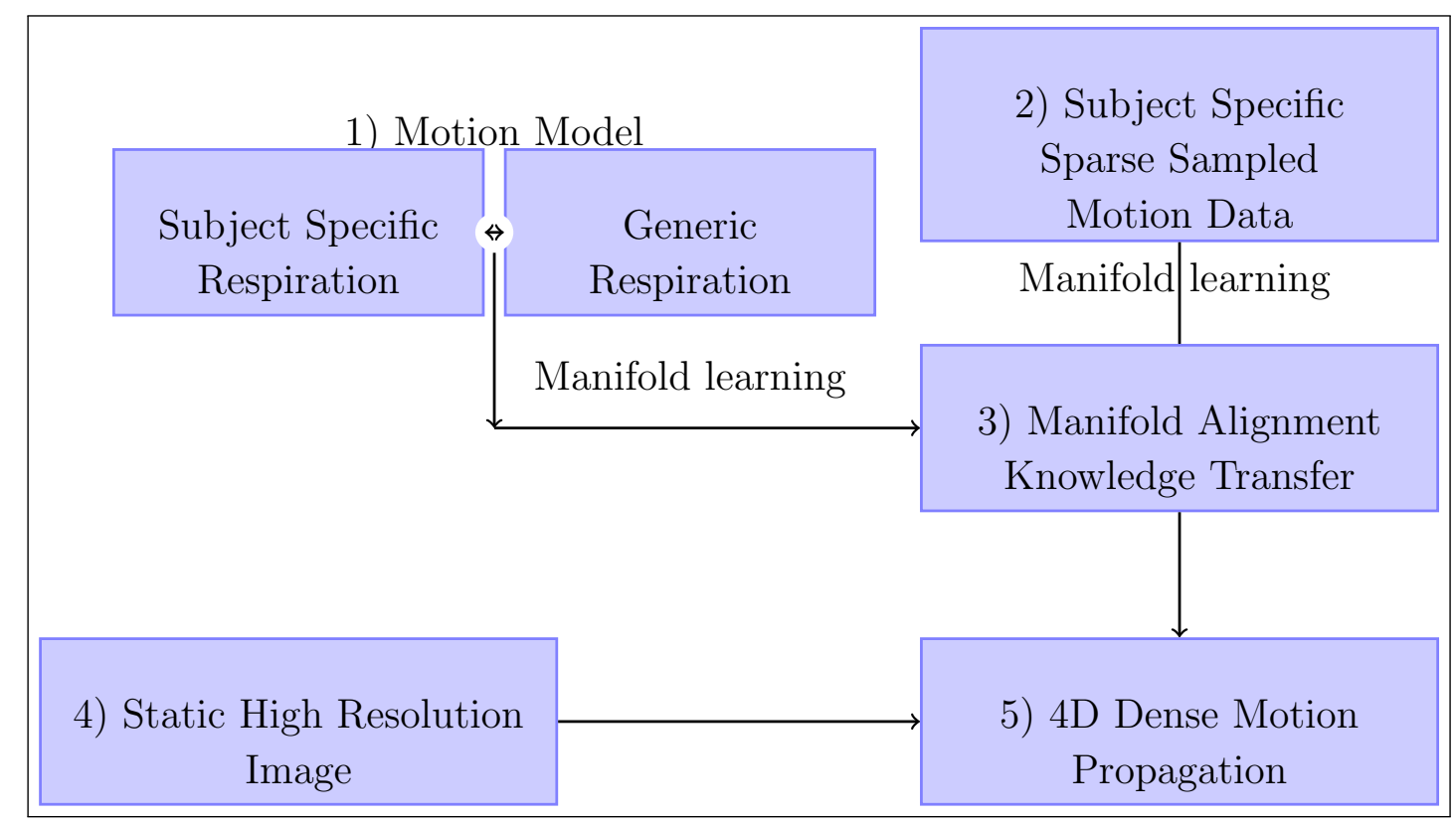

Figure 1: Overall framework for dense motion propagation from sparse samples, using manifold learning and alignment

\section{Theory and Framework}

\subsection{Manifold Learning and $P C A$}

We start our description of the framework that we propose by providing a brief overview of the theory of manifold learning, and our use of PCA to compactly describe abdominalthoracic respiratory motion. We then describe the overview of our approach and how manifold alignment links to both the dense and sparse motion models that represent the inputs to the approach and provide a framework to estimate full motion fields from sparse samples which are then used to propagate a static high resolution image. The generic framework proposed consists of five key steps (see figure 1): [1] The method starts with a motion model, which in this work is a subject specific model but may be extended in future work to a generic model. For the particular problem used here, this is a single average respiratory cycle representation of the motion. This is aligned with a set of sparse samples of free breathing respiratory motion [2], the sparse samples may for example be image projections of full volumes or partial imaging data such as individual slices. Sparse data may also originate from a different imaging source used to construct the model in [1]. 3] This alignment may take place in the image domain, as often occurs in image registration methodology, or in an alternative space. For the work described here, this alignment takes place in eigen space. The alignment allows knowledge from 
one domain to be transferred to the other. Thus sparse motion estimates during free breathing may be used to construct dense motion fields [5] to propagate a static high resolution image[4]. (See figure 1).

Respiratory motion models are commonly constructed to estimate the complex deformation in the abdominal thoracic cavity from surrogate signals of respiration. Non-rigid image registration (Avants, Epstein, Grossman and Gee 2008) can be used to provide accurate voxel-wise deformations between image volumes at different phases of a respiratory sequence. Dimensionality reduction or manifold learning techniques attempt to embed such high dimensionality data into a lower dimensional space whilst preserving its characteristic properties. The ordered parameters in this lower dimensional domain permit a more compact but accurate representation of whole body motion. Numerous manifold learning or dimensionality reduction techniques exist such as laplacian eigenmaps, isomaps, local linear embedding, multi-dimensional scaling etc. (Maaten et al. 2008). However, in this work we choose the simplest form, Principal Component Analysis (PCA). The high dimensional respiratory motion data that we observe can then be approximated by a linear manifold in eigen-space (Smith et al. 2013a) and so provides a straightforward linear projection of the data onto the principal components which form the basis vectors of the new linear subspace.

\subsection{Dense Motion Model}

PCA which is performed on a training data set consisting of a single average re-binned respiratory cycle thus provides a basis for the manifold which describes the characteristic properties of respiratory motion. This densely sampled dynamic motion may be drawn from a patient specific representation of motion or a more generic description to be used as a global model. In mathematical terms PCA is used in this work on the vector fields as a result of registration whereby a set of concatenated $3 \mathrm{D}$ vector components $x, y$ and $z$ are derived from registration of $\mathbf{K}$ image volumes with $M$ voxels back to a reference frame such that $\mathbf{v}_{k}=\left[\boldsymbol{v}_{1, x, \mathbf{k}}, \boldsymbol{v}_{1, y, \mathbf{k}}, \boldsymbol{v}_{1, z, \mathbf{k}}, \ldots, \boldsymbol{v}_{\mathbf{M}, x, \mathbf{k}}, \boldsymbol{v}_{\mathbf{M}, y, \mathbf{k}}, \boldsymbol{v}_{\mathbf{M}, z, \mathbf{k}}\right]^{T} \in R^{n}$ with $n=3 . M$. The training class may thus be defined as $\mathbf{V}=\left[\mathbf{v}_{1}, \mathbf{v}_{2}, . ., \mathbf{v}_{k} . ., \mathbf{v}_{\mathbf{K}}\right]$, mean centering results in $\mathbf{X}$ as defined below:

$$
\begin{aligned}
& \mathbf{x}_{k}=\mathbf{v}_{k}-\overline{\mathbf{v}}, \quad \text { where } \overline{\mathbf{v}}=\frac{1}{K} \sum_{k=1}^{K} \mathbf{v}_{k}, \\
& \text { thus } \mathbf{X}=\left[\mathbf{x}_{1}, \mathbf{x}_{2}, \ldots, \mathbf{x}_{k} . ., \mathbf{x}_{\mathbf{K}}\right] \in \mathcal{R}^{n \times K}
\end{aligned}
$$

PCA learns a linear model from subsequently calculating the covariance matrix $\mathbf{C}$ (equation 3) and finds a linear combination of the original variables such that the covariance matrix is diagonal. The diagonalization of $\mathbf{C}$ can be achieved by singular value decomposition (SVD) of $\mathbf{X}$ (equation 4).

$$
\begin{aligned}
& \mathbf{C}=\frac{1}{K} \mathbf{X X}^{T}=\frac{1}{K} \sum_{j=1}^{K} \mathbf{x}_{j} \mathbf{x}_{j}^{T} \in \mathcal{R}^{n \times n} \\
& \mathbf{C}=\mathbf{U} \cdot \operatorname{diag}\left(\sigma_{i}^{2}\right) \cdot \mathbf{U}^{T}
\end{aligned}
$$


The columns $\mathbf{u}_{i}$ form a set of orthogonal eigenvectors $\mathbf{U}=\left[\mathbf{u}_{1}, \mathbf{u}_{2}, \ldots, \mathbf{u}_{i} . ., \mathbf{u}_{K-1}\right]$ whilst $\sigma_{1} \geq \sigma_{2} \ldots \geq \sigma_{K-1}$ are the standard deviations within the data along each ordered eigenvector. By virtue of mean centering the span of the dataset and rank of $\mathbf{X}$ and $\mathbf{C}$ is at most $J=K-1$. The eigenvectors thus diagonalize the covariance matrix and may be used as a basis for a lower dimensional manifold. The result of the projection into the lower dimensional domain is a set of uncorrelated weights $\mathbf{w}_{k}=\left[\mathbf{w}_{1}, \mathbf{w}_{2}, \ldots, \mathbf{w}_{j} . ., \mathbf{w}_{\mathbf{J}}\right]$ or scores allowing any particular instance, $\mathbf{v}_{k}$, of the motion vector field to be described as shown in equation(5). PCA thus restricts solutions or motion estimates to be a linear combination of eigenvectors, whilst $U^{T}$ provides a basis that spans the columns of $\mathbf{X}$. Any new estimate made of $\mathbf{w}$ by design results in an instance of $\mathbf{x}$ that is a linear combination of previous examples contained in $\mathbf{X}$. This results in motion estimates to be made that are within the span of the eigenvectors; gross motion such as jerks or shifts may prove problematic to account for using this approach. But this is not inconsistent with other approaches to motion correction within the literature (Baumgartner et al. 2017, King et al. 2012).

$$
\mathbf{w}_{k}=\mathbf{U}^{T} \mathbf{x}_{k} \quad \mathbf{x}_{k}=\mathbf{U} \mathbf{w}_{k} \quad \mathbf{v}_{k}=\overline{\mathbf{v}}_{k}+\sum_{\mathbf{j}=1}^{\mathbf{J}} \mathbf{w}_{\mathbf{j}_{(k)}} \mathbf{u}_{j}
$$

A property of PCA is the assumed Gaussian probability density for $\mathbf{w}$ which is utilized further in section 2.4 .

$$
P(\mathbf{w})=2 \pi^{-K / 2} e^{\frac{1}{2}\|\mathbf{w}\|^{2}}
$$

The principal components thus provide an orthogonal basis for the smallest linear space containing the manifold. With knowledge of the linear subspace the weights or loadings $\mathbf{w}_{k}$ allows a simpler representation of the underlying motion and lends structure to the high dimensional data. In this work we propose to learn the eigenbasis of respiratory motion using an average fully sampled $(3 \mathrm{D}+\mathrm{t})$ respiratory cycle constructed by rebinning numerous phase gated 2D slices. We subsequently refer to this dataset as 'the average dynamic'. The eigenbasis thus provides a mapping function from the original data to the latent space in the manifold. This a-priori knowledge allows us to obtain full motion estimates from partial sampled motion data as described below. The utilization of such sparse motion has previously been proposed (Smith et al. 2013b, Mcclelland et al. 2014) and provides a practical expedient to estimate full field of view free-breathing motion. We propose that motion vector fields from sparsely acquired sagittal slices whilst a volunteer/patient is free breathing may be considered to be sub samples from the manifold derived from the average dynamic dataset. However it is important to note that the properties of the sparsely sampled data maybe disparate from the average dynamic with both possibly containing different image features. This is more-so when sparse imaging data is derived from a different source or imaging modality such as ultrasound. We therefore utilize manifold alignment coupled with the approach adopted by (Blanz et al. 2002) to give full deformation field estimates from sparse samples as 


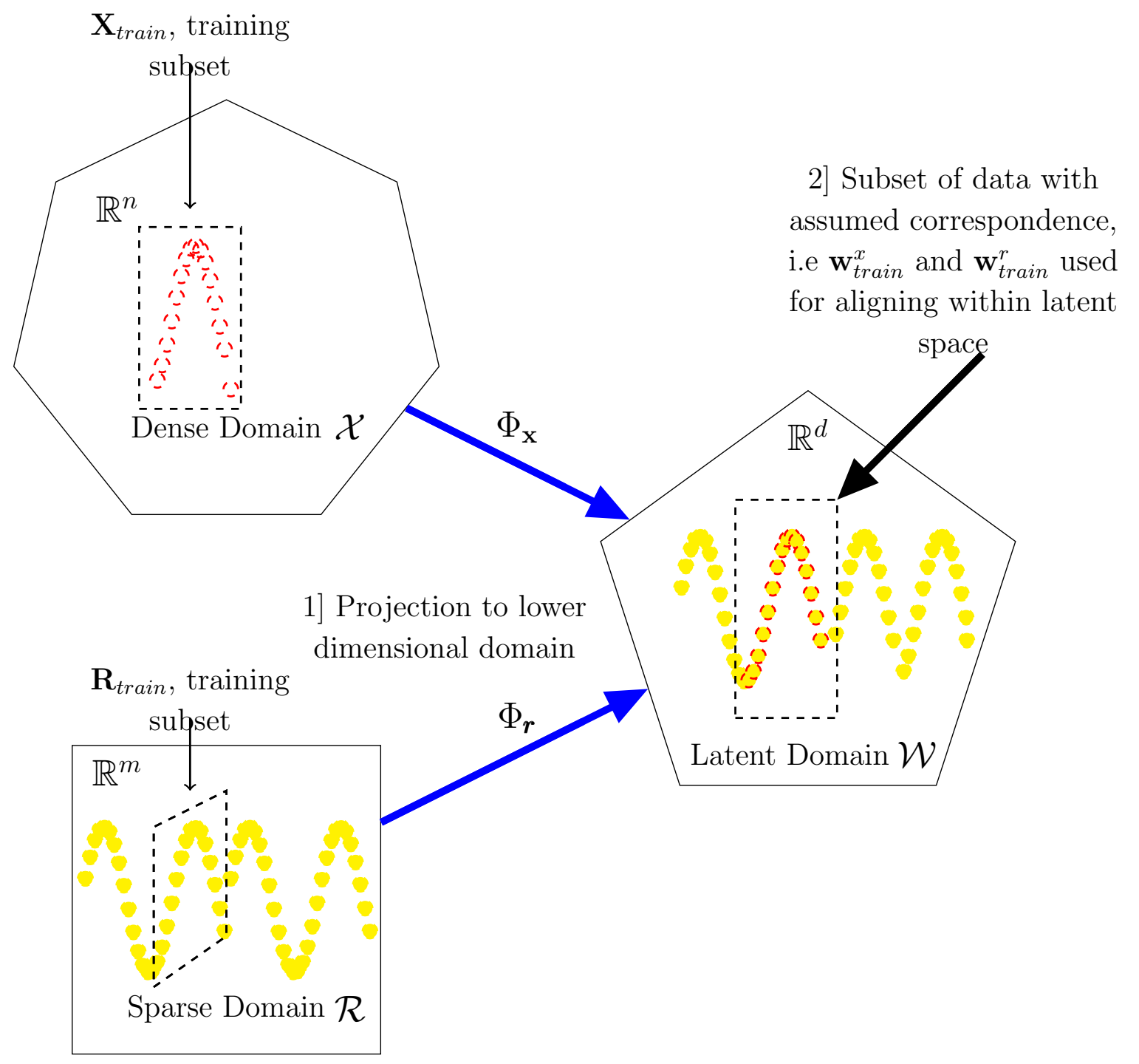

Figure 2: Schematic of manifold alignment using a two step process.[1] Both sparse and dense data in $\mathcal{R}$ and $\mathcal{X}$ respectively are projected independently into separate lower dimensional domains. [2] A pairwise correspondence from a subset of each data set in the latent domain $\mathbf{w}_{\text {train }}^{x}$ and $\mathbf{w}_{\text {train }}^{r}$ are used to find an alignment between the two separate lower dimensional domains. In this work Procrustes alignment aligns the sparse lower dimensional embeddings to the dense lower dimensional embeddings in $\mathcal{W}$.

described in section (2.4). A relevant approach to estimate full motion from sparse samples has been reported in Preiswerk et al. (2012). However our work differs in a key important aspect: We remove the need to manually define motion points in the image domain by labelling image features of the sparse and fully sampled data, instead we utilizing manifold alignment to allow a generalizable framework across different image sources which exhibit heterogeneous features. To further explain this point and for completeness a brief overview of manifold alignment follows. 


\subsection{Manifold Alignment}

Manifold alignment builds mappings between two or more disparate datasets by aligning their underlying manifolds and provides a geometric framework for knowledge transfer across data sets. As described in figure 2 , given two dataset $\mathbf{X}=\left[\mathbf{x}_{1}, \mathbf{x}_{2}, . ., \mathbf{x}_{k} . ., \mathbf{x}_{\mathbf{K}}\right]$ with $\mathbf{x}_{k} \in \mathbb{R}^{n}$ and $\mathbf{R}=\left[\mathbf{r}_{1}, \mathbf{r}_{2}, . ., \mathbf{r}_{k} . ., \mathbf{r}_{\mathbf{K}}\right]$ with $\mathbf{r}_{k} \in \mathbb{R}^{m}$ with a subset of assumed known pairwise correspondence, manifold alignment aims to determine an optimal map between $\mathbf{X}$ and $\mathbf{R}$ and hence from one domain to the other, i.e $\mathcal{R} \rightarrow \mathcal{X}$. New instances of $\mathbf{r}_{k}$ thus allow estimates within domain $\mathcal{X}$ to be made. This fundamental property thus allows the mapping of partial samples from one domain to articulate samples from another domain. Within medical imaging this might represent partial samples within the same modality or, between modalities such as partial information from ultrasound or fluoroscopy being used to derive plausible motion in MRI/CT or other tomographic data. Manifold alignment alleviates the need to determine complex mappings in the high dimensional image domain directly and instead aims to find an optimal alignment by projecting the data into a latent or lower dimensional domain $\mathcal{W}$ with $\mathbf{w}_{k} \in \mathbb{R}^{\mathbf{d}}$. This alignment may be achieved in a one or two step process. With a one step process the projections into the lower dimensional domain, $\Phi_{\mathbf{x}}$ and $\Phi_{\mathbf{r}}$ also provide an embedding which results in correspondence in the latent space. A two step process however (figure 2) firstly projects each input data set into a lower dimensional space independently using any variety of dimensionality reduction techniques; followed by a linear manifold alignment on the embedded data, holding one data set fixed and mapping each additional data set onto the fixed manifold. The alignment is performed on a subset of data in which pairwise correspondence is assumed. For the purposes of respiratory motion modelling this may be a pre-determined number of phases within a single respiratory cycle from the dense and sparse data or, as in this case, a single respiratory state such as the beginning of inhale.

$$
\begin{aligned}
& \Phi_{x}: \mathbb{R}^{\mathbf{n}} \rightarrow \mathbb{R}^{\mathbf{d}} \\
& \Phi_{r}: \mathbb{R}^{\mathbf{m}} \rightarrow \mathbb{R}^{\mathbf{d}}
\end{aligned}
$$

One of the simplest manifold alignment algorithms is Procrustes alignment (Wang and Mahadevan 2008) whereby a subset of the lower dimensional embeddings (labelled train) $\mathbf{W}_{\text {train }}^{x}=\Phi_{x} \mathbf{X}_{\text {train }}$ and $\mathbf{W}_{\text {train }}^{r}=\Phi_{r} \mathbf{R}_{\text {train }}$, in which correspondence is assumed, are translated such that their centroids are at the origin. The problem is then simplified to finding an orthonormal rotation matrix $\mathbf{Q}$ and a scaling factor $\mathbf{h}$ such that $\left\|\mathbf{W}_{\text {train }}^{x}-\mathbf{h} \mathbf{W}_{\text {train }}^{r} \mathbf{Q}\right\|_{F}$, known as the Frobenius norm, is minimized. The optimal solution is given by the singular value decomposition (SVD) of $\mathbf{W}_{\text {train }}^{r T} \mathbf{W}_{\text {train }}^{x}$ (equations $8-10)$

$$
\begin{aligned}
& S V D\left(\mathbf{W}_{\text {train }}^{r T} \mathbf{W}_{\text {train }}^{x}\right)=U \Sigma V^{T} \\
& \mathbf{Q}=U V^{T} \\
& \mathbf{h}=\frac{\operatorname{trace} \Sigma}{\operatorname{trace}\left(\mathbf{W}_{\text {train }}^{r T} \mathbf{W}_{\text {train }}^{r}\right)}
\end{aligned}
$$


When using PCA as the dimensionality reduction technique the individual mappings $\Phi_{\mathrm{x}}$ and $\Phi_{\mathrm{r}}$ are derived from the eigenvectors of the covariance matrices of $\mathbf{X}$ and $\mathbf{R}$ respectively. The learnt mappings allow instances within the dense domain $\mathbb{R}^{\mathbf{n}}$ to be estimated from the partial, or sparse sampled domain $\mathbb{R}^{\mathrm{m}}$ and hence estimates of full motion (i.e, 3D+t) to be derived from sparse motion (e.g., 2D+t). In this work we use a Bayesian model similar to (Preiswerk et al. 2012) used in the liver. However the new work is applied to the entire abdominal-thoracic cavity, and, with Procrustes alignment providing knowledge transfer from one domain to another removes the need for manual interaction with the image data. Moreover, this can be seen to render the approach generalizable and robust to inter modality / inter patient whole body motion estimation. We assess its performance in a patient specific case to demonstrate the improved performance produced when incorporating manifold or Procrustes alignment. This is in comparison to when a Bayesian model alone is used, as the manifold alignment stage provides the mechanism to estimate internal motion more accurately when it is sampled from a disparate source, such as an external surrogate signal or sparse samples of internal motion. The framework is described in further detail below.

\subsection{Sparse Motion Model}

Instances of sparse $2 \mathrm{D}$ motion $\mathbf{r} \in \mathbb{R}^{\mathrm{m}}$ may be concatenated in a similar manner described in section 2.1 to produce a reduced number of features of full 3D motion $\mathbf{v} \in \mathbb{R}^{\mathbf{n}}$. We assume that $\mathbf{r}$ is related to $\mathbf{v}$ by a linear mapping $H$, such as the product of a projection that selects a subset of components from $\mathbf{v}$ (equation 11).

$$
\begin{aligned}
& H: \mathbb{R}^{\mathbf{n}} \rightarrow \mathbb{R}^{\mathbf{m}} \quad \text { with } \mathbf{n}<\mathbf{m} \\
& \mathbf{r}=H \mathbf{v}
\end{aligned}
$$

With the assumption that $H$ is known precisely, mean centering gives the following error function $\mathbf{y}$ (equation 12).

$$
\mathbf{y}=r-H \overline{\mathbf{v}}=H \mathbf{x}
$$

where $\overline{\mathbf{v}}$ represents full $3 \mathrm{D}$ motion vector fields. A unique solution to $H$ is unlikely to exist, therefore a vector $\mathbf{x}$ that minimizes $E(\mathbf{x})=\|\mathbf{H} \mathbf{x}-\mathbf{r}\|^{2}$ is sought. To reduce the number of free parameters we restrict any new instance of $\mathbf{x}$ to be a linear combination of previous examples and hence within the span of $\mathbf{X}$. This is achieved by defining the reduced version $\mathbf{U}^{\prime}$ of the full eigenvectors $\mathbf{U}$, i.e $\mathbf{U}^{\prime}=\left[\mathbf{u}_{1}^{\prime}, \mathbf{u}_{2}^{\prime}, \ldots, \mathbf{u}_{j}^{\prime} . ., \mathbf{u}_{\mathbf{J}}^{\prime}\right]$. The projection into the latent space using the reduced eigenvectors will thus result in a submanifold constrained within the original manifold. The reduced eigenvectors correspond to the index value of the sparse measurement within the fully sampled deformation field. One thus seeks model parameters that minimize $E\left(\mathbf{w}_{k}\right)=\left\|\mathbf{U}^{\prime} \mathbf{w}_{k}-\mathbf{r}_{k}\right\|^{2}$. The optimum model $\mathbf{w}_{k}$ may be found by utilizing the SVD to determine the pseudo-inverse denoted as *. 


$$
\begin{aligned}
& \mathbf{U}^{\prime}=\mathbf{S A V}^{T} \\
& \mathbf{U}^{\prime *}=\mathbf{V A}^{*} \mathbf{S}^{T} \\
& \mathbf{w}_{k}=\mathbf{U}^{\prime *} \mathbf{r}
\end{aligned}
$$

From a manifold alignment perspective the above notion assumes $\Phi_{x}=\mathbf{U}$ and $\Phi_{r}=\mathbf{U}^{\prime}$. The above assumption however is only valid if both $\mathbf{r} \in \mathbb{R}^{\mathbf{m}}$ and $\mathbf{x} \in \mathbb{R}^{\mathbf{n}}$ are homogeneous with non-disparate manifolds. This assumption is inaccurate and becomes particularly so when $\mathbf{r}$ is derived from a different imaging source than $\mathbf{x}$ as they will contain different image features and image quality. In this case a manifold alignment step is required as novel valid estimates of $\mathbf{x}$ are required. We incorporate Procrustes alignment to map the manifold $\mathcal{R} \rightarrow \mathcal{X}$. Equation 13 thus becomes 14, with $\mathbf{w}_{\text {train }}^{r}$ and $\mathbf{w}_{\text {train }}^{x}$ being matched respiratory phases of correspondence in the partial image and dense image domain respectively.

$$
\mathbf{h w}_{k} \mathbf{Q}=\mathbf{U}^{\prime *} \mathbf{r}
$$

where:

$$
\begin{aligned}
& S V D\left(\mathbf{w}_{\text {train }}^{r}{ }^{T} \mathbf{w}_{\text {train }}^{x}\right)=U \Sigma V^{T} \\
& \mathbf{Q}=U V^{T} \\
& \mathbf{h}=\frac{\operatorname{trace} \Sigma}{\operatorname{trace}\left(\mathbf{w}_{\text {train }}^{r}{ }^{T} \mathbf{w}_{\text {train }}^{r}\right)}
\end{aligned}
$$

A Bayesian approach is taken to avoid over fitting, and to also permit the model to generalize to novel values and account for any wrong assumptions on $\mathbf{U}^{\prime}, \mathbf{h}$ and $\mathbf{Q}$. A full derivation is given in Appendix A. An expression (17) for the likelihood of observing the measured vector $\mathbf{r}_{k}$ can be obtained, assuming it is subject to uncorrelated Gaussian noise with a variance $\sigma_{N}^{2}$. Utilizing Bayes' rule and equation 6 then the probability of model parameters given the observed data is obtained (18).

$$
\begin{aligned}
& P\left(\mathbf{r} \mid \mathbf{r}_{\text {model }}\right) \propto e^{\frac{1}{2 \sigma_{N}^{2}}\left\|\mathbf{r}_{\text {model }}-\mathbf{r}\right\|^{2}} \\
& P(\mathbf{r} \mid \mathbf{w}) \propto \quad e^{\frac{1}{2 \sigma_{N}^{2}}\left\|\mathbf{U}^{\prime} \mathbf{w}-\mathbf{r}\right\|^{2}} \\
& P(\mathbf{w} \mid \mathbf{r}) \propto \quad e^{\frac{1}{2 \sigma_{N}^{2}}\left\|\mathbf{U}^{\prime} \mathbf{w}-\mathbf{r}\right\|^{2}} \cdot e^{\frac{1}{2}\|w\|^{2}}
\end{aligned}
$$

As the model parameters sought are those in the domain of the dense field we add an additional Procrustes alignment step (equation 19) with $w^{\prime}=h w Q$.

$$
P\left(w^{\prime} \mid r\right) \propto e^{\frac{1}{2 \sigma_{N}^{2}}\left\|U^{\prime} h w Q-r\right\|^{2}} e^{\frac{1}{2}\|h w Q\|^{2}}
$$

If the sparse samples were already extracted from the domain of the dense field this would result in a unity transform, i.e $w^{\prime}=w$. Taking the logarithm of equation 19 allows the definition of the cost function (equation 21).

$$
\begin{aligned}
& E=-2 . \log P\left(w^{\prime} \mid r\right) \\
& E=\frac{1}{\sigma_{N}^{2}}\left\|U^{\prime} w-r\right\|^{2}+\left\|w^{\prime}\right\|^{2}+\text { const }
\end{aligned}
$$


An analytical method to minimize the cost function may be found and is expanded upon in Appendix A. A regularization factor $\eta=\sigma_{N}^{2} \geq 0$ is introduced which acts as a trade off between prior probability of the solution and matching quality of the model fit, which in the presence of noise in $r$ or wrong assumptions of $U^{\prime}$ may result in heavily distorted solutions $v_{k}$. With appropriate choice of $\eta$, SVD of the reduced eigenvectors and Procrustes analysis to find values of $h$ and $Q$, then an optimal value of $w$ and hence $x$ may be found (equation 22).

$$
x=U h\left(V \operatorname{diag}\left(\frac{a_{i}}{a_{i}^{2}+\eta}\right) S^{T} r\right) Q
$$

Using the above, the imaging framework for the methodology is described below.

\subsection{Imaging Framework}

Using equation 22 and the aforementioned 2-step alignment process, we now put forward an imaging framework to demonstrate a novel robust and efficient method of recovery of full 3D motion from sparse samples using three imaging datasets. In this exemplar illustration we use: a high resolution static volume, an average re-binned single respiratory cycle, herein referred to as the average dynamic, and a sparse-sampled free-breathing dataset. Utilizing an eigenbasis derived from the average dynamic, full motion estimates are made from the sparsely sampled free breathing dataset which is used to propagate or "animate" the high resolution static volume. For initial spatial correspondence the average dynamic and sparse datasets are transformed to the coordinate system of the static volume using a composition of image registrations. We utilize a symmetric diffeomorphic image registration with cross correlation followed by a diffeomorphic Demons using a multi resolution approach (Avants et al. 2008)(Advanced Normalization Tools ( ANTS ) 2014). This results in both forward $(\rightarrow)$ and backward $(\leftarrow)$ warping fields. The set of transformations corresponding to the $K$ phases of the average dynamic dataset from the average dynamic reference frame, Vref defined at end exhale are described by $T_{\text {Dyn } \leftarrow \text { Vref }}$. Similarly, for the sparse data set, we use $N=8$ sagittal slices for the $T$ phases seen during free breathing. These are registered to a sparse reference frame, $V r e f^{\prime}$, defined at end exhale resulting in transformations $T_{\text {sag } N \leftarrow \text { Vref' }}$ (equation 23).

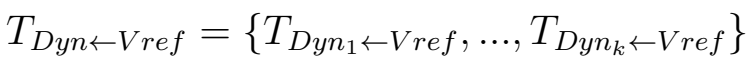

$$
\begin{aligned}
& T_{\text {sagN } \leftarrow \text { Vref }}=\left\{T_{\text {SagN }_{1} \leftarrow V r e f^{\prime}}, \ldots, T_{\text {SagN }_{T} \leftarrow V r e f^{\prime}}\right\}
\end{aligned}
$$

The full set of dynamical sagittal transformations consisting of all 8 slices is thus described by $T_{\text {sag } \leftarrow \text { Vref }}=\left\{T_{\text {sag } 1}, T_{\text {sag } 2}, \ldots T_{\text {sag } N}\right\}$. Affine transformations from the reference phase of the average dynamic dataset, and the sparse sagittal dynamic data set, to the high resolution static volume $V_{o}$ are then found $\left(T_{D y n_{V r e f}} \leftarrow V_{o}\right.$ and $T_{\text {sag }_{V r e f} \leftarrow V_{o}}$ respectively). Composition of these transformations allows the entire set of average dynamic and sparse dynamic transformations to be expressed in the co-ordinate system 
of the static volume (equation 24)can hence be used to describe a fully sampled estimate of the free breathing motion vector field.

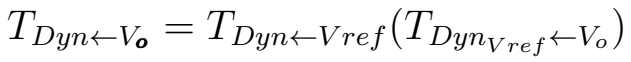

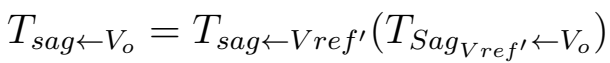

However the transformations $T_{s a g \leftarrow V_{o}}$ are, spatially, not fully sampled. In order to address this issue the statistical properties of the average dynamic samples $T_{\mathbf{D y n} \leftarrow V_{o}}$ are exploited to estimate the missing transformation components using the method described in section 2.4. Figures 3-5 provide a schematic summary of the proposed imaging framework. Frames within the average re-binned respiratory cycle are registered back to a common reference frame defined at the beginning of inhale (figure 3). Subsequent 2D registrations (figure 4) of the sparse samples whilst the volunteer is breathing continuously allows calculation of the sparse motion estimates. The sparse motion model (see section 2.4) is then utilized to estimate fully sampled free breathing motion, constrained within the eigenbasis. The resulting 4D motion vector fields may then be used to articulate, or animate, a corresponding high resolution static volume (figure 5).

\section{Method}

Evaluation tests were performed on two datasets (A and B) and described in sections III-A and III-B respectively. Testing on dataset(A) was to evaluate the method with full $4 \mathrm{D}$ motion vector fields available for ground truth comparison therefore allowing a quantitative measure of effectiveness. Testing on dataset (B) was to demonstrate the result of sparse sampling and static image articulation.

\subsection{Evaluation with Dynamic 3D MRI}

Validation tests were performed using fully sampled dynamic 4D MRI data (Tsoumpas et al. 2011) from three volunteers with voxel resolution $1.48 \times 1.48 \times 5.5 \mathrm{~mm}$ (r-l, supinf, ant-post) and temporal resolution with each image volume acquired every 0.7 seconds. Each volunteer dataset consisted of 35 image volumes, thus encompassing 24.5 seconds of free breathing. However, the high temporal resolution of the image volumes results in poor contrast noise and Gibbs ringing artefacts. These were reduced using anisotropic diffusion filtering in a similar manner to Buerger et al. 2011. This results in smoother homogeneous image regions that contain little structural information but avoids smoothing between different tissue types, thus preserving edges and serves as a ground truth dataset in this work. Internal phases were grouped into cycles dependent upon the inflection points of externally observed chest motion extracted from the MRI dataset. The mean position of the externally observed chest surface served as the external surrogate signal. This was extracted from the MRI dataset using simple thresholding. One single respiratory cycle was utilized to build the eigenbasis (analogous to the average re-binned cycle). Eight sagittal slices (30mm apart) (figure 4) for all 
internal phases of the data were extracted to represent a sparse sampled dataset, and utilized with the sparse motion model to estimate full vector fields. Procrustes alignment was performed using the sparse slices and volumes from the training respiratory phases. The reference frame of the average dynamic was propagated with estimates of the full field. As the fully sampled data provides a ground truth representation, the root mean square (rms) error of the estimated motion vector fields obtained from deformable registration can be used for evaluation. Note, these are calculated across the entire image volume, rather than within select ROIs, in order to determine the robustness of the approach across a variety of different spatio-temporal motion distortions within the complete abdominal-thoracic cavity. Three models were tested for comparison, namely: (i) the Bayesian model, (ii) Procrustes alignment, and (iii) the Bayesian model with Procrustes alignment incorporated.

\subsubsection{Evaluation with Dynamic 3D MRI Using An External Surrogate Signal}

To evaluate the generalizability of the framework to different surrogate signals of internal motion; which for example may become available during PET/MR imaging (e.g. navigator and / or external monitoring) a validation test was performed for one volunteer using extracted chest motion; which replaces the sparsely sampled MRI. The surrogate external signal was formed by simulating an orthogonal projection of the anterior chest surface extracted from each phase of MRI data which serves as a pseudo depth measurement and now represented by $\mathbf{R}=\left[\mathbf{r}_{1}, \mathbf{r}_{2}, . ., \mathbf{r}_{k} . ., \mathbf{r}_{\mathbf{K}}\right]$ with $\mathbf{r}_{k} \in \mathbb{R}^{m}$. The reduced version of the eigenvectors $\mathbf{U}^{\prime}$ now become the eigenvectors derived from mean centred chest motion during the single training cycle. The surrogate domain however is now more disparate than when utilizing sparse internal samples of motion. Estimates of internal motion are again derived using three models namely: (i) the Bayesian model alone, (ii) Procrustes alignment, and (iii) the Bayesian model with Procrustes alignment incorporated; a comparison is also made to when an internal surrogate signal, i.e sparse internal samples are used.

\subsection{Testing with $M R I$}

Testing with MRI was performed on an exemplar single volunteer. The datasets consisted of, 1] A high resolution static volume with voxels of dimensions $3 \times 1.5 \times 1.5 \mathrm{~mm}$. 2) A single average respiratory cycle produced from an average re-binned dynamic sequence with 21 sagittal slices, voxel dimension $3 \times 3 \times 3 \mathrm{~mm}$, averaged over $12 \mathrm{~mm}$. 3) Fifty phases of sparse dynamics, consisting of 8 sagittal slices, 3x6x6mm, 24mm apart (r-l,sup-inf, ant-post). Each sparse dynamic free breathing volume had a temporal sampling rate of 1.2 seconds for each set of 8 spare sampled slices. The sparse dynamic data corresponding to the beginning of inhale was used with the beginning of inhale phase from the average dynamic to determine a manifold alignment using Procrustes analysis. The sparse motion model was used to estimate full vector fields from sparse dynamic slices. The high resolution static was then propagated with motion estimates from sparse samples (figure 5). 


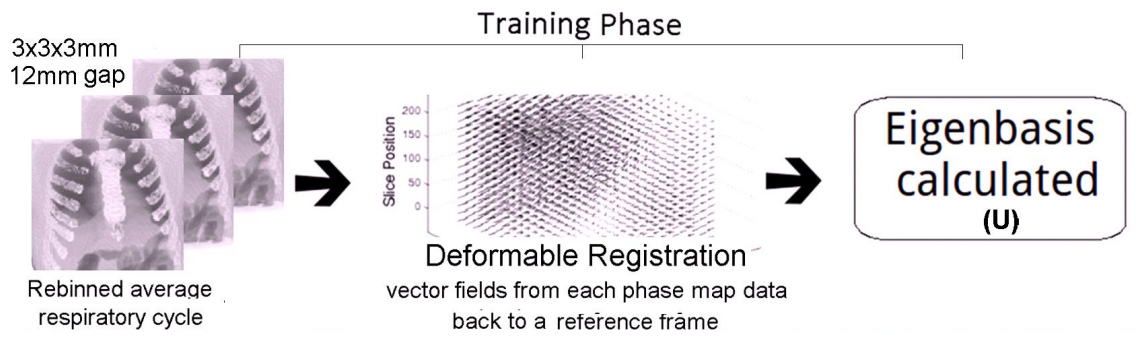

Figure 3: A set of vector fields from symmetric registration back to a reference frame of multiple phases are calculated from a single re-binned average respiratory cycle, adopted from Smith et al. (2013b). This provides an eigenbasis for new estimates of full vector fields from sparse samples as described in figure 4.

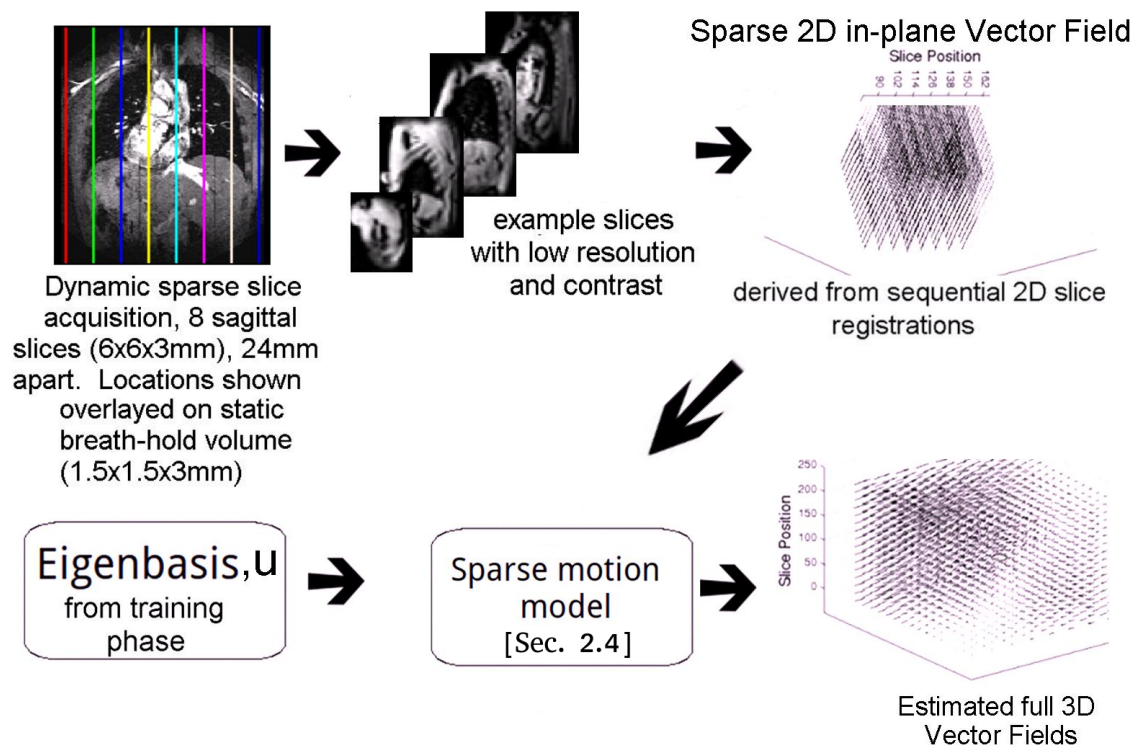

Figure 4: Registration of sparse 2D dynamic slices to a reference frame when patient is free breathing provide a subset of full 3D vector fields. The sparse motion model (section 2.4) allows estimation of novel full 3D vector fields with motion modes derived from the sparse samples when the patient is breathing continuously.
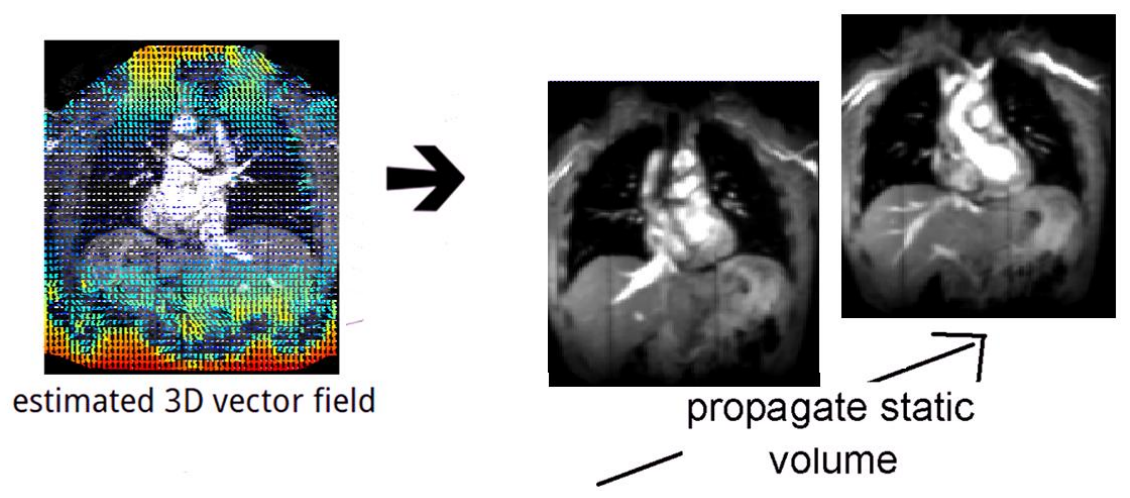

Figure 5: Estimated full 3D vector fields, constrained by an eigenbasis allowing propagation of the high resolution static dataset 


\section{Results \& Discussion}

A comparison was made of the performance of the three aforementioned models. Figure 6 compares the weights of the first PCA loading using the three models versus that of the ground truth when sparse samples of the internal motion are used as the surrogate signal. A distribution of weights in the Bayesian models is present as one increases the value of $\eta$. This distribution results in numerous realizations of weights for the Bayesian and Bayesian with Procrustes model (figure 6) thus the dashed lines actually appear as thicker more dense lines due to this distribution. For clarity a zoomed in version is displayed in figure 7 demonstrating an example of such a distribution. With each iter-
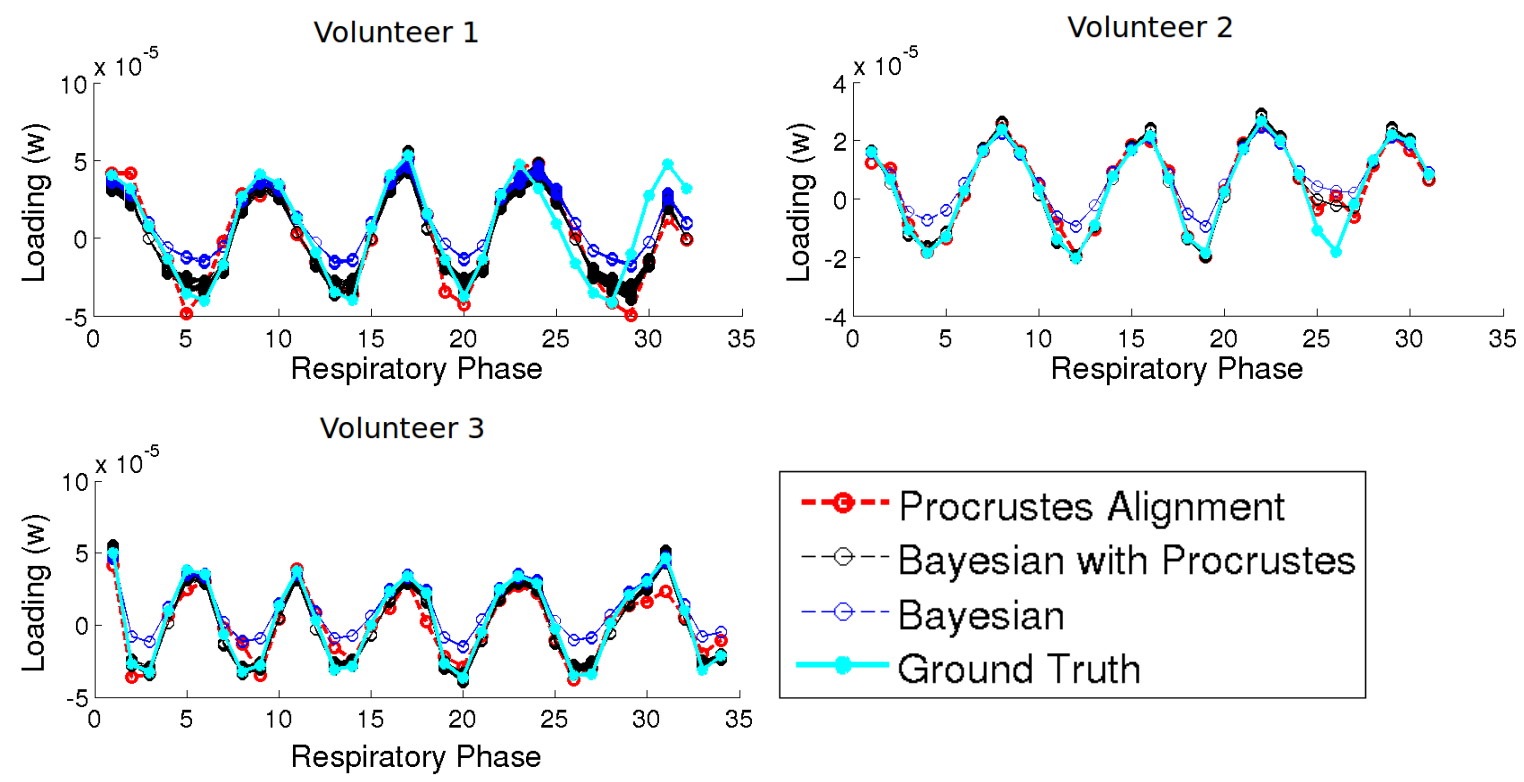

Figure 6: A comparison of first PCA loadings for three volunteers using the three models also displayed is the ground truth.

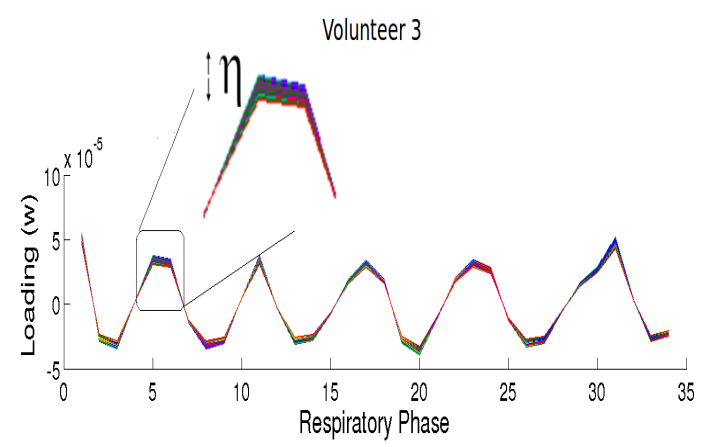

Figure 7: Example of distribution of estimated PCA loadings for the first component with iterations of $\eta$ for the Bayesian with Procrustes alignment model. The value of $\eta$ was varied from 0 to 1000000 , with each iteration resulting in perturbations of the estimated loadings. 
ation the probability of the estimated loadings is maximized, this results in a reduction in reconstructed error of the vector fields. Figure 8 demonstrates the rms voxel error for the Bayesian model with Procrustes alignment as a function of $\eta$ for all three volunteers. An optimal value for $\eta$ was chosen as that which is observed in volunteer 1 , however $\eta$ did not vary significantly when the analysis was repeated on data for volunteer 2 and 3 . In practice a judicious choice of $\eta$ should be made with training data available for the imaging modality / surrogate signals being utilized.
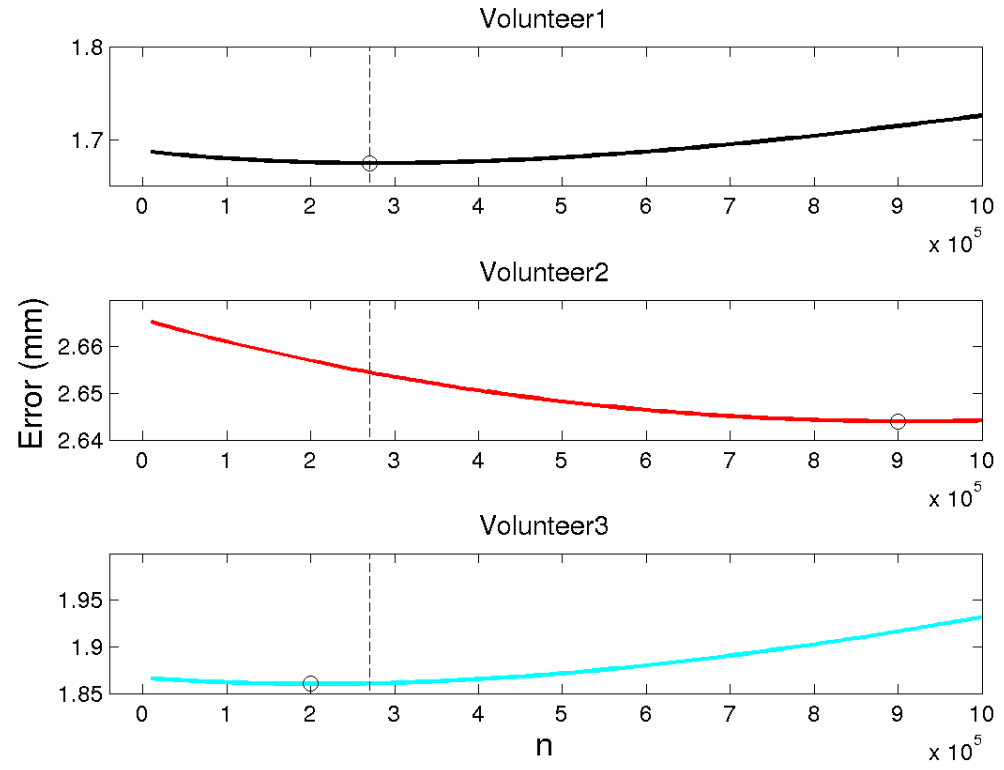

Figure 8: Root mean square error of estimated vector fields for Procrustes Bayesian model in comparison to ground truth data with increasing values of $\eta$ for all three volunteers. The dashed line highlights the choice of $\eta$ which gives the minimum error in volunteer 1 , which was chosen arbitrarily.

To evaluate the framework when utilizing a different external surrogate signal, volunteer 1 was chosen. Figure 9 plots a comparison of the first PCA loadings estimated for this volunteer when using the external surrogate signal with the three models. As observed the Bayesian model alone without the inclusion of Procrustes analysis makes poor estimates of the ground truth PCA loadings; with the inclusion of Procrustes to align these disparate manifolds estimates become closer to the ground truth. Figure 10 demonstrates the rms voxel error for the Bayesian model with Procrustes alignment as a function of $\eta$ for volunteer 1 whilst using the external surrogate signal versus the internal surrogate signal. The optimal value for $\eta$ which was originally chosen for volunteer 1 using the internal surrogate signal is highlighted. It is evident that the internal surrogate signal produces better estimates of internal motion than the external surrogate signal. A comparison was made of the mean rms error for all respiratory phases for each model when using an external surrogate signal (figure 11). The choice of $\eta$ when using the external surrogate signal was chosen as the value that minimizes the error 

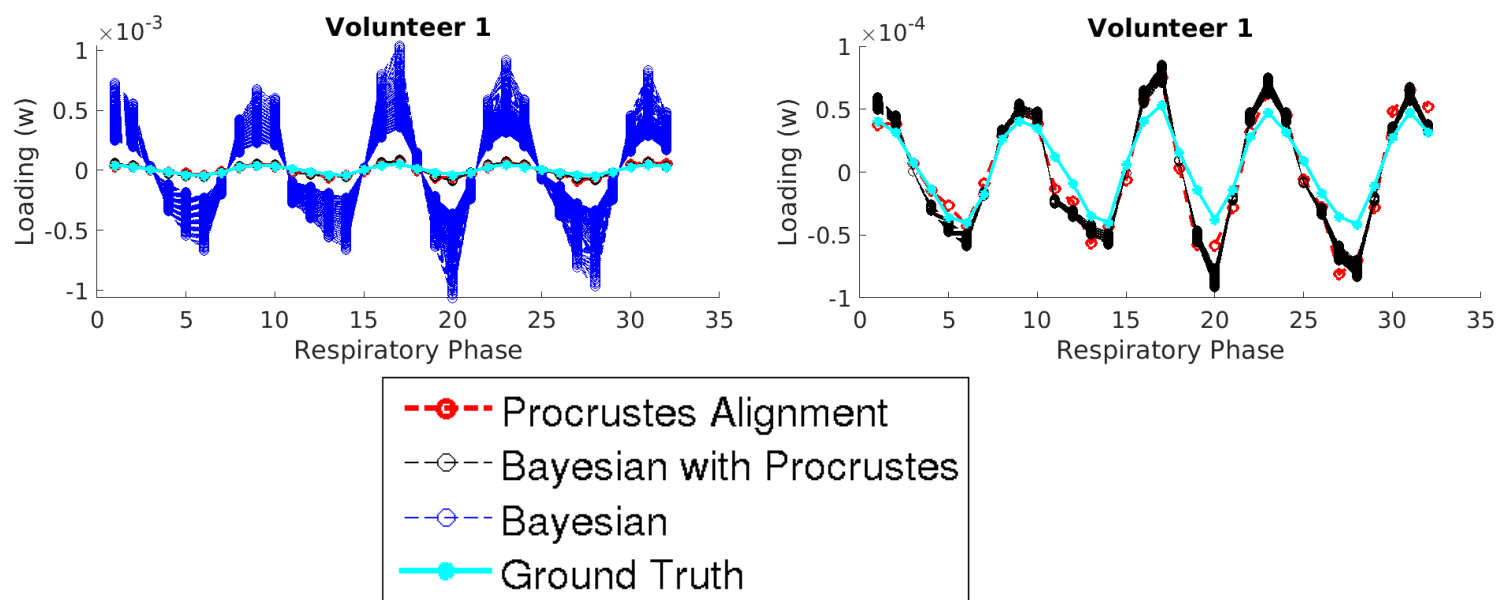

Figure 9: A comparison of first component PCA loadings for one volunteer using an external surrogate signal to estimate internal motion using the three models also displayed is the ground truth. As can be seen as the external surrogate signal is disparate from the internal manifold the estimates of motion made using the Bayesian model alone are inaccurate (left diagram) and become far more accurate and closer to the ground truth when a Procrustes alignment step is introduced (right diagram).

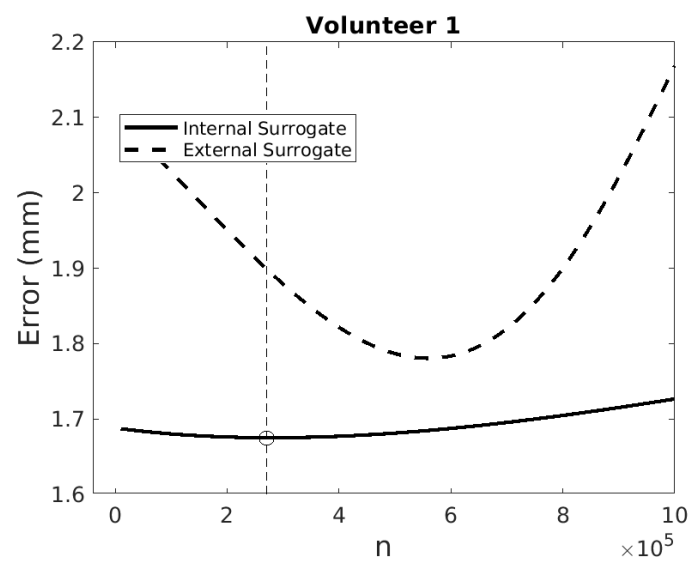

Figure 10: Mean root mean square error of estimated vector fields for the Procrustes Bayesian model in comparison to ground truth data with increasing values of $\eta$ for volunteer 1. A comparison is made when estimates of motion are found using the internal surrogate signal versus the external surrogate signal. The dashed line highlights the choice of $\eta$ which gives the minimum error in volunteer 1 when using the internal surrogate signal

in this volunteer. Even with this biasing of the result figure 11 demonstrates the poor performance of the Bayesian model alone, as the external surrogate signals manifold is disparate to the internal. Procrustes alignment however is effective at aligning these manifolds and the Bayesian model with Procrustes alignment incorporated provides the most accurate model as shown in figure 11. It is noteworthy however that the internal 
surrogate signal still out performs the external surrogate signal in this case. This highlights clearly the advantage of our proposed framework over the Bayesian sparse model used by Preiswerk et al. (2012); the incorporation of the manifold alignment stage not only alleviates the requirement to manually define motion points in the two disparate domains (i.e fully sampled motion and sparse motion) but also allows the application of other surrogate signals to derive estimates of motion highlighting a further advantage over recently proposed respiratory motion models (Baumgartner et al. 2017). During the model application stage, the surrogate signal (internal or external, from varying sources) may be used to estimate dense motion fields allowing free breathing respiratory motion estimates to be made; this alleviates the stringent requirement of re-binning data into one average respiratory cycle.

A comparison was subsequently made of the mean rms error for all respiratory phases for each model when using the internal surrogate signal for all three volunteers and is highlighted in figure 12. This demonstrates the increased performance of the Bayesian model with Procrustes over the Bayesian model alone and Procrustes alignment alone. The Bayesian model with Procrustes alignment demonstrated a 7.6\%, 20.5\% and 21.9\% reduction in error for each volunteer respectively, with an average reduction of $\approx 16 \%$ in comparison to using the Bayesian model alone.

We now more closely inspect the effect of the framework for all three volunteers whilst using the internal surrogate signal. Figure 13 demonstrates the location of the sparse samples and the reconstructed error for each individual cycle using the Bayesian model with Procrustes alignment with an error of $\approx 2 \mathrm{~mm}$, validating the approach. Figure 14 presents the distribution of error magnitude for volunteer 1 for the phase of motion with (a) the maximum mean error and (b) the minimum mean error. As can be seen the majority of error values fall below $\approx 2 \mathrm{~mm}$. An image of the error magnitude for this volunteer is also presented (figure 14 (c)) highlighting the largest error in the region of the diaphragm therefore (figure $14(\mathrm{~d})$ ) tracks the position of the left and right diaphragm for both the ground truth dynamic imaging data and the imaging data modelled using the Bayesian model with Procrustes alignment. Tracking was performed using the position of the maximum image gradient for each phase of motion at the location of the left and right diaphragm. As can be observed the modelled position of the diaphragm matches closely the ground truth data with only small perturbations noticed. The increased error magnitude demonstrated in these regions may be due to artefacts from, ghosting and Gibbs ringing confounding the accuracy of modelled motion estimates. Figure 15 calculates the error of the right and left diaphragm modelled using the Bayesian with Procrustes alignment for each volunteer and each individual cycle with a mean error being of the order of $\approx 3 \mathrm{~mm}$.

Figure 16 plots the maximum error magnitude in millimetres for the three volunteers versus respiratory phase with each individual cycle highlighted. No evident regions of respiratory phase demonstrate a persistently maximum error signal. With optimum 


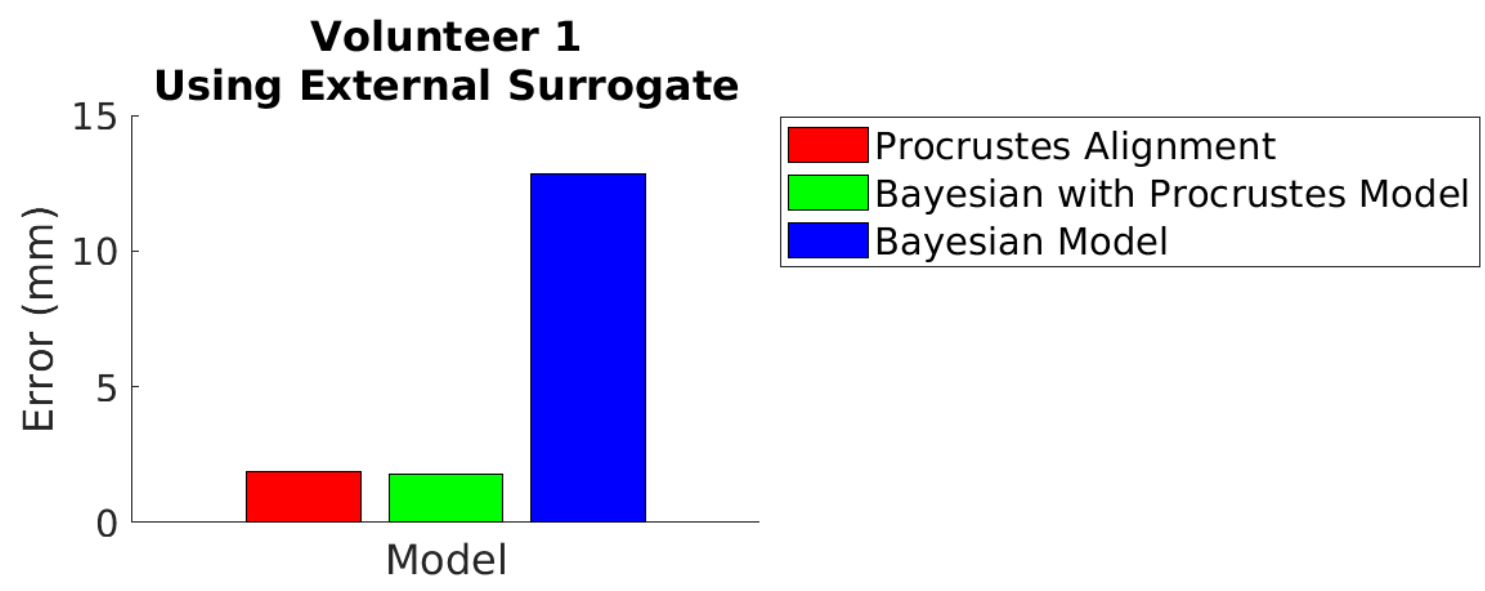

Volunteer 1

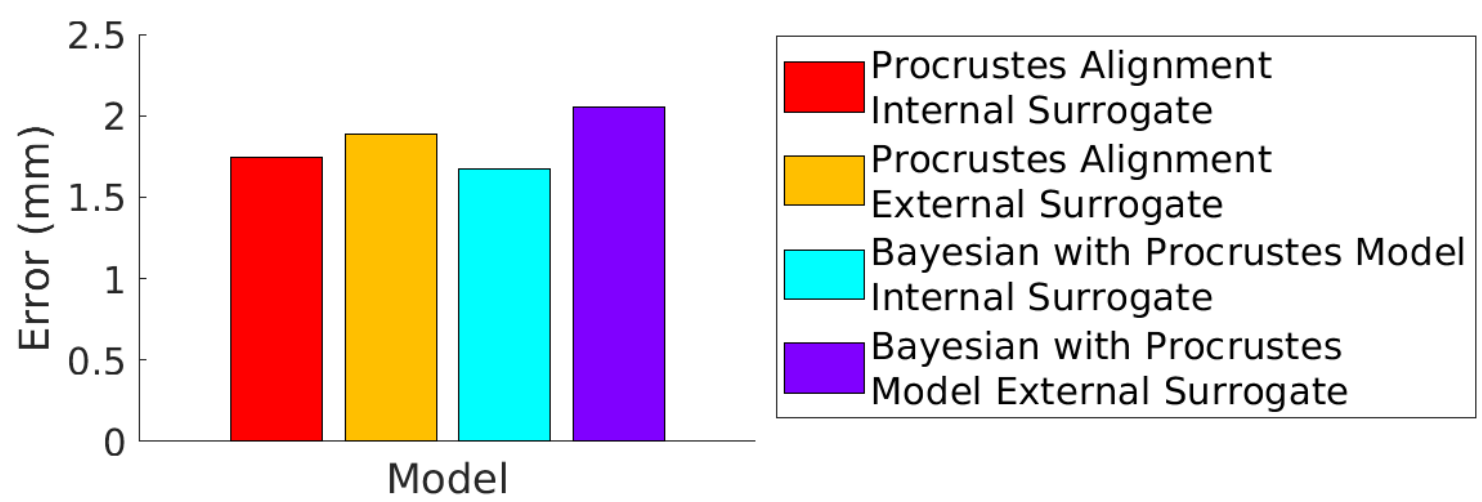

Figure 11: a) Mean root mean square error of estimated deformation fields vs. ground truth deformation fields for three models tested for volunteer 1 when using the external surrogate signal, as shown the Bayesian model alone performs poorly in comparison to when the manifolds are aligned with the Procrustes step. b) Comparison of the mean r.m.s.e for of the estimated deformation fields vs. the ground truth deformation fields when varying the surrogate signal (internal and external). A comparison is made of two models Procrustes alignment, and Bayesian with Procrustes alignment. As can be observed greater accuracy is obtained when using the internal surrogate signal in comparison to the external surrogate signal (intra model comparison); the Bayesian model with Procrustes alignment achieves the greatest accuracy of $1.6 \mathrm{~mm}$; whilst utilizing the internal surrogate using this model, versus the external surrogate results in a reduction in error of 18 percent. 

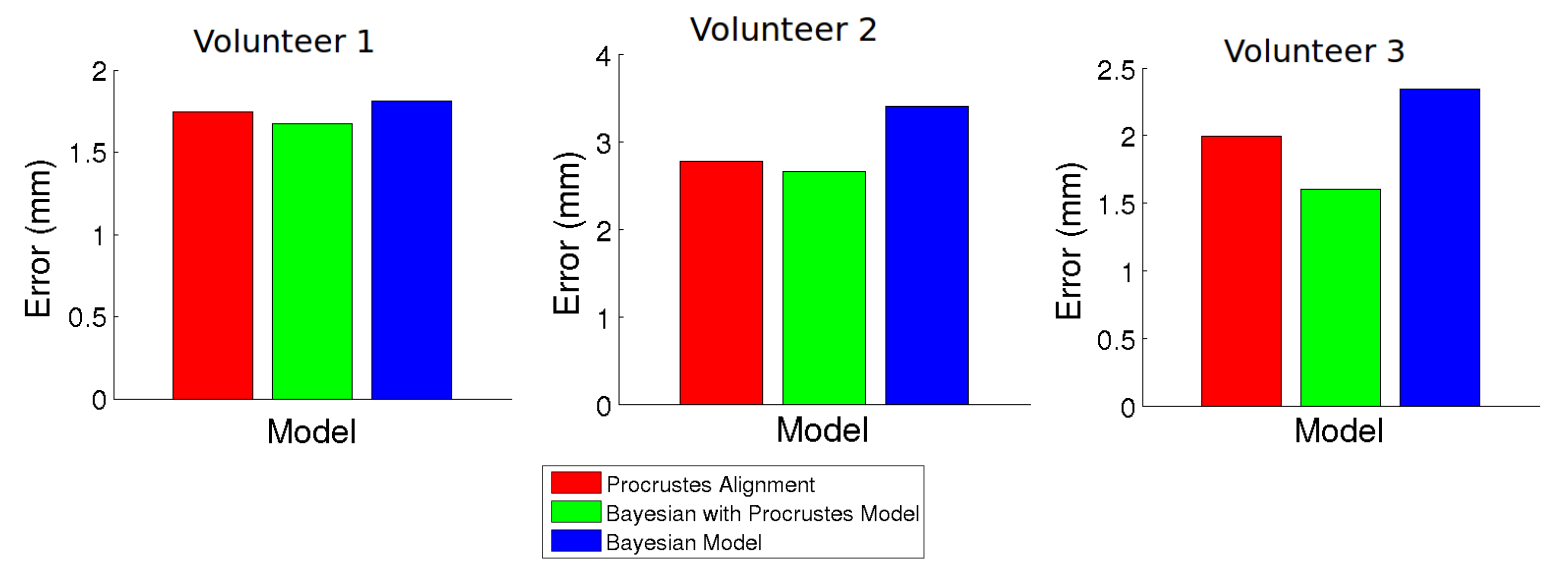

Figure 12: Mean root mean square error of estimated deformation fields vs. ground truth deformation fields for three models tested for all three volunteer datasets when using the sparse MRI as the surrogate signal of motion.
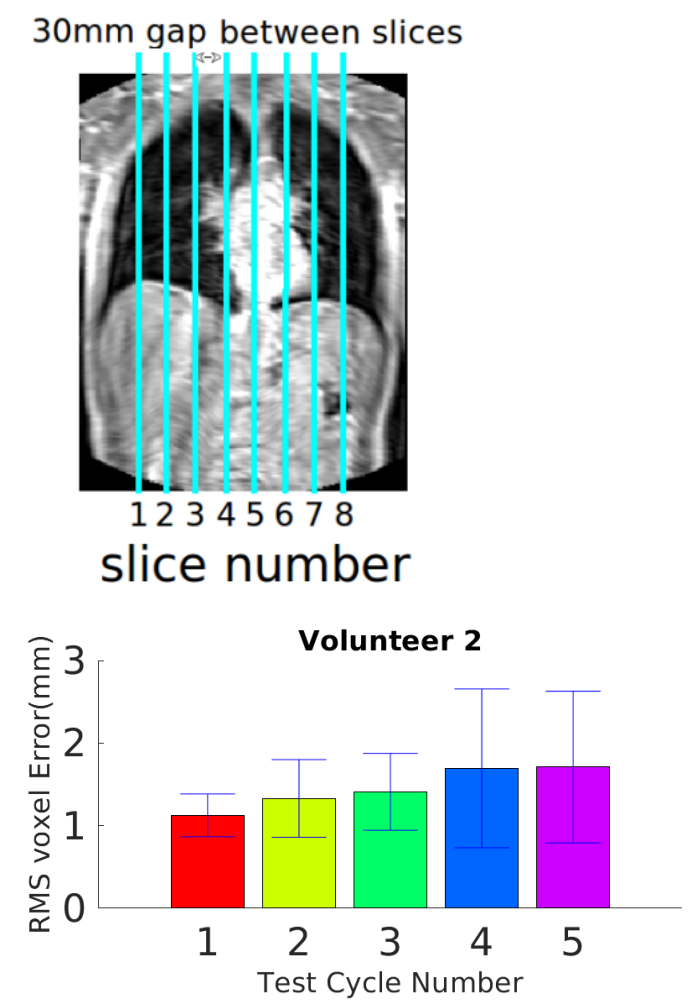
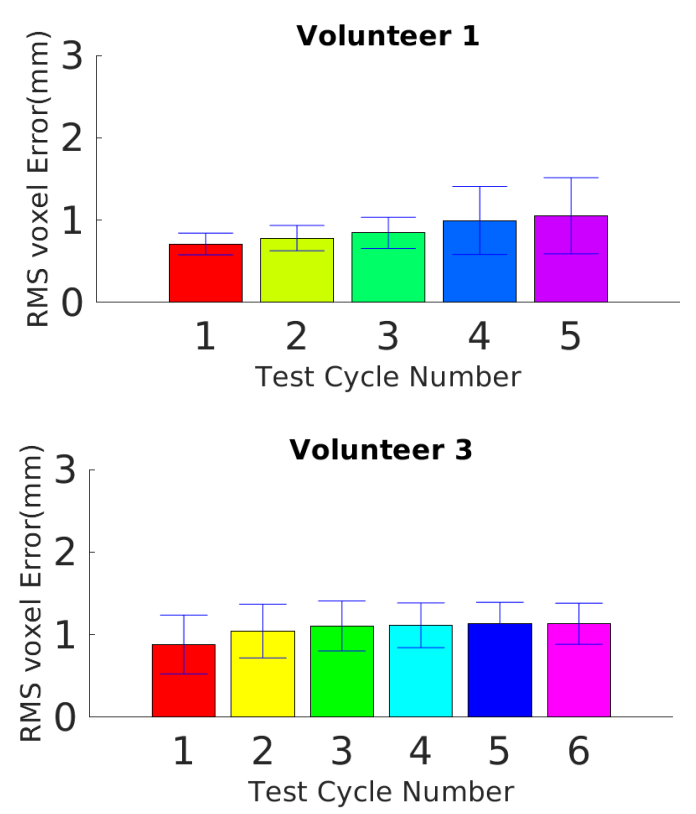

Figure 13: Upper left shows location of sparse slices from which full motion is estimated, remaining bar charts show root mean square error of the estimated versus the actual vector field averaged for each individual respiratory cycle for each volunteer over the entire field of view.

performance the Procrustes Bayesian model was used to estimate full vector fields from sparse samples in the testing dataset (section 3.2). Figure 17 compares the PCA loadings along the first principal component of the re-binned average dynamic data with a single 
(a)

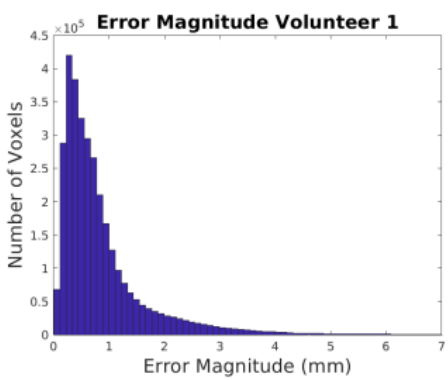

(b)
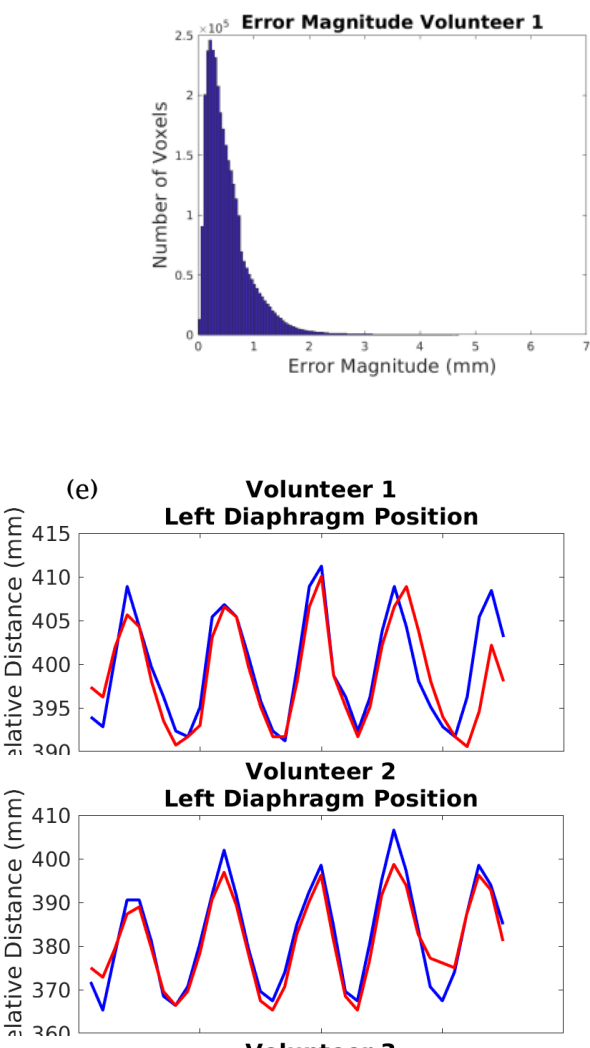

Volunteer 3

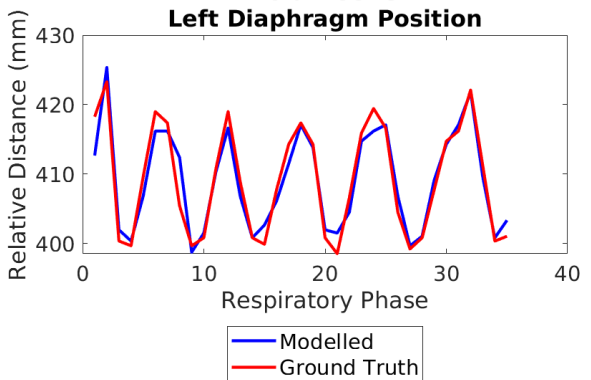

(c)

Displacement

Error Magnitude (mm)

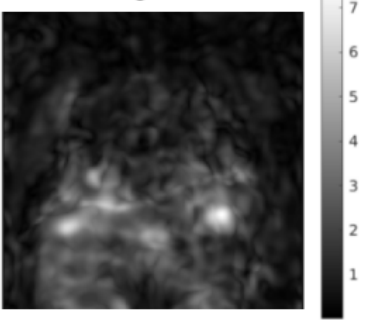

(d)
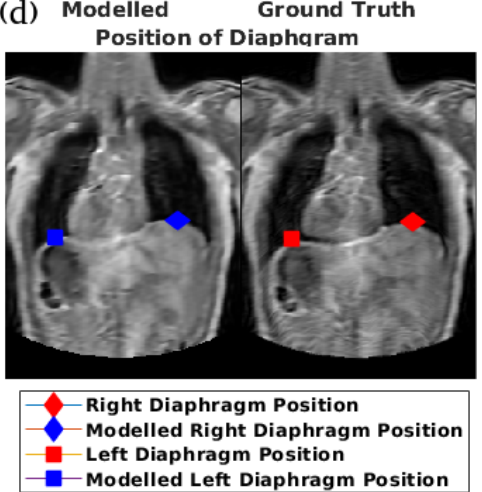

Left Diaphragm Position

Volunteer 1

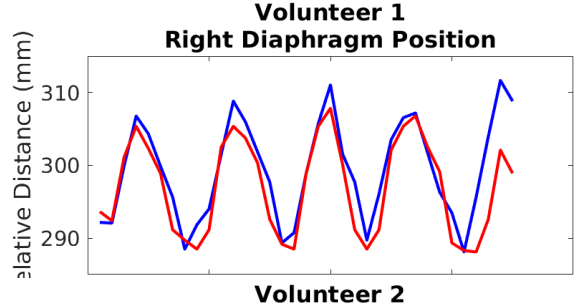

Right Diaphragm Position

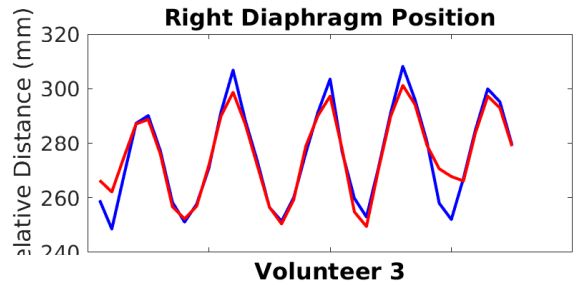

Right Diaphragm Position

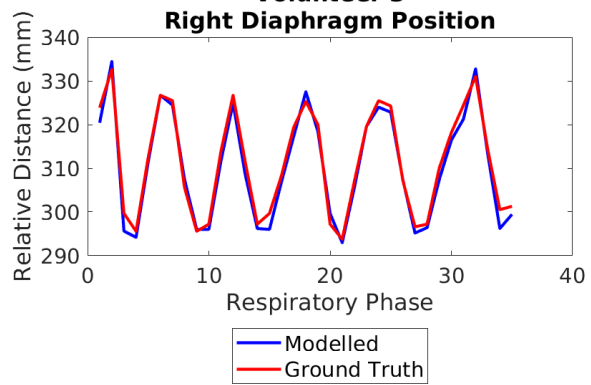

Figure 14: (a) Histogram presenting the distribution of error magnitude (mm) for volunteer 1 for the phase in the sequence that demonstrates the maximum mean error $(\approx 7 \mathrm{~mm})$, the distribution highlights the majority of voxels have an error magnitude $<2 \mathrm{~mm}$. (b)

Histogram presenting the distribution of error magnitude ( $\mathrm{mm}$ ) for volunteer 1 for the phase in the sequence that demonstrates minimum mean error; the majority of voxels have an error magnitude $<1 \mathrm{~mm}$. (c) Error magnitude of a single slice with the highest error magnitude in volunteer 1; areas of increased error are located near the left and right diaphragm. (d) The location of the left and right diaphragm tracked using the position of the maximum gradient in these regions for the modelled and ground truth dynamic images. (e) The location of the right and left diaphragm for the modelled (blue) versus the ground truth image (red) highlighting the small perturbations of the modelled location from the ground truth. 

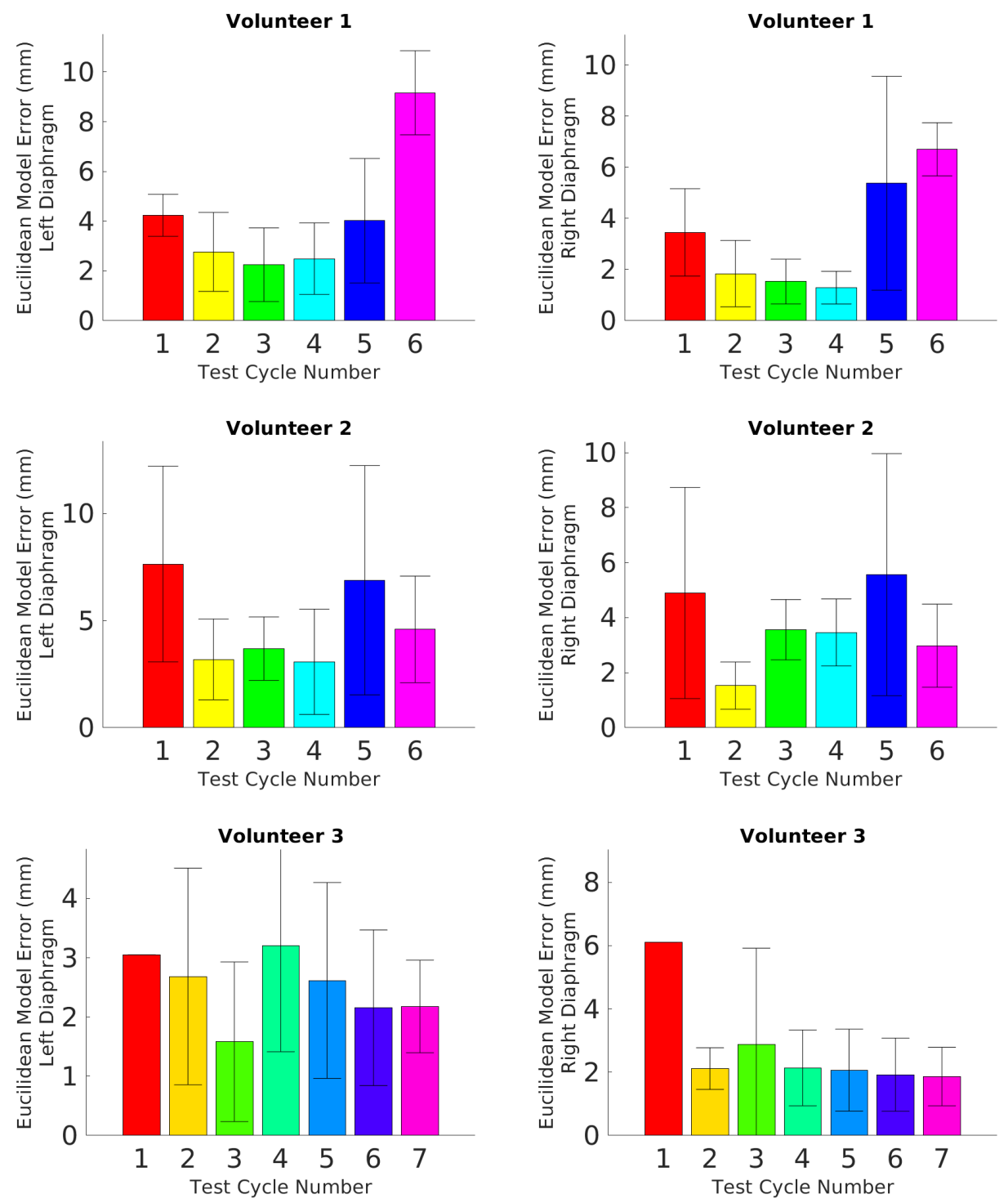

Figure 15: Mean Euclidean model error ( $\mathrm{mm}$ ) for the left (left column) and right (right column) diaphragm for all three volunteers, with the average error being taken over individual cycles. Error bars represent the standard deviation of the error within each cycle.

cycle and those estimated using the sparse motion model in the testing MRI dataset. The PCA loadings offer a lower dimensionality representation of global internal motion. As highlighted the sparse motion (red trace) demonstrates numerous respiratory cycles with varying duration and amplitude in comparison to the single cycle of the average dynamic (black trace). By constraining the sparse motion estimates with the eigenbasis of the average dynamic new realizations of dense vector fields lie in the span of the average dynamic allowing plausible motion to be determined. Moreover the framework in its current state uses sagittal slices to estimate dense motion and full vectors from sparse samples. Example sagittal images of the propagation of the static reference 

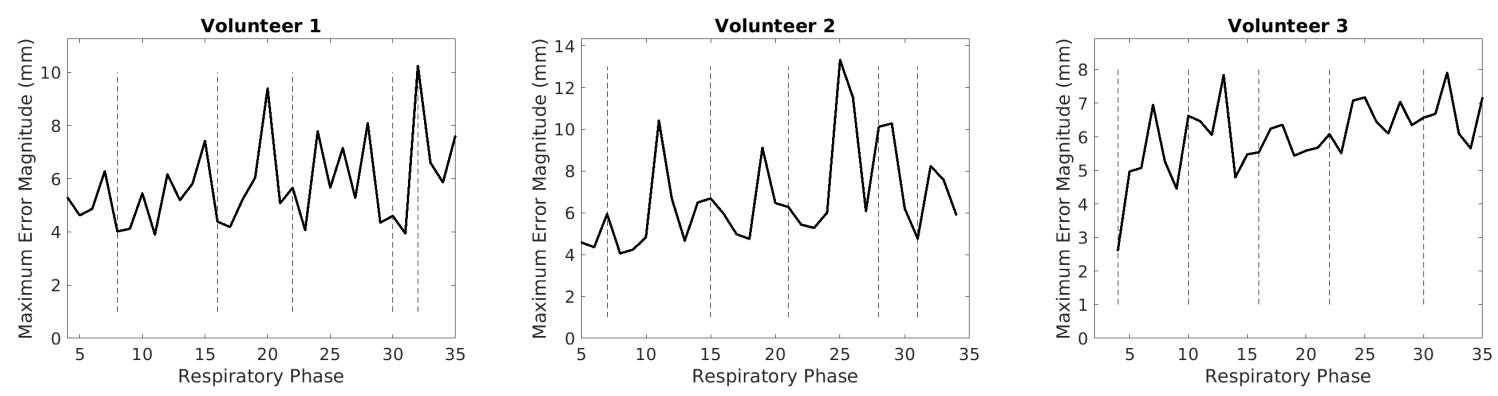

Figure 16: Maximum magnitude error for all three volunteers versus respiratory phase, dashed lines highlight individual cycles, maximum error of $10.25 \mathrm{~mm}, 13.3 \mathrm{~mm}$ and $7.9 \mathrm{~mm}$ for volunteers 1-3 respectively; no evident region of respiratory phase demonstrates a persistently increased maximum error.

(a)

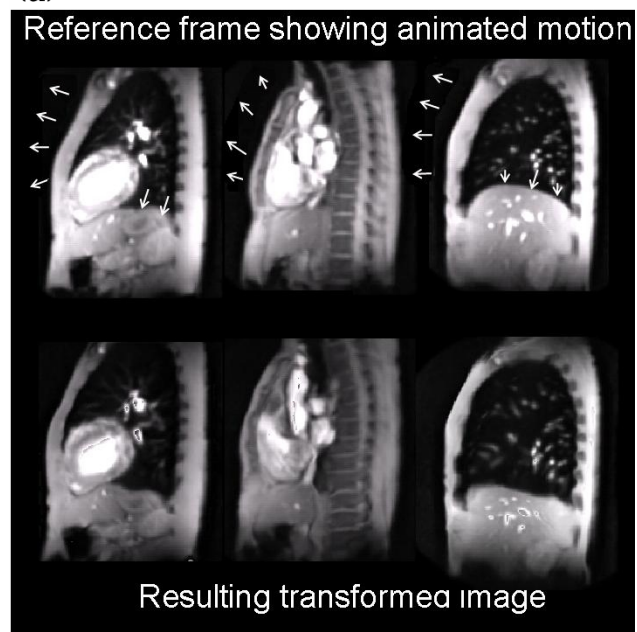

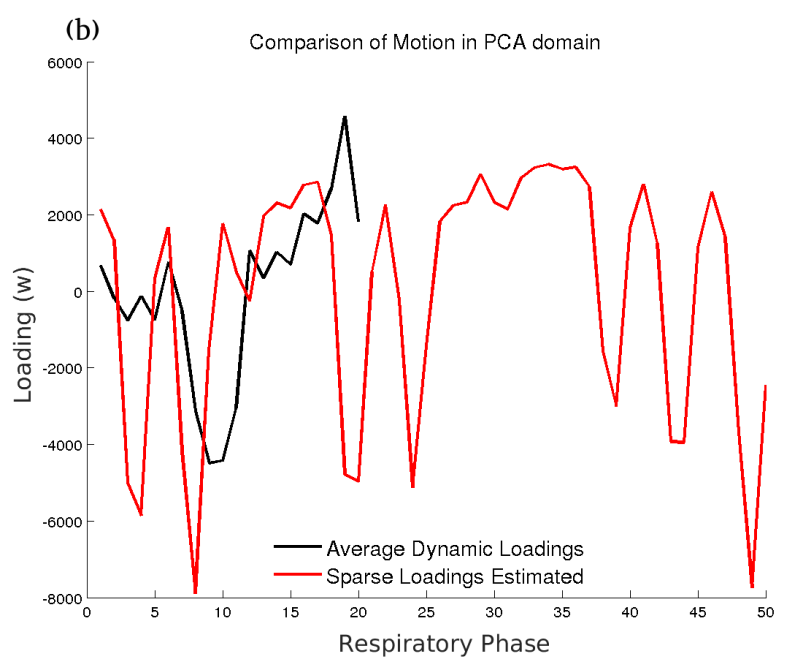

Figure 17: (a) Exemplar image quality of a static reference frame propagated with dense displacement field estimated from sparse samples. (b) A comparison of the PCA loadings along the first principal component for the average dynamic (black) and estimated PCA loadings using the sparse motion model (red) highlighting the varying nature of motion captured during sparse respiration in comparison to the single average dataset.

volume from sparse samples is demonstrated at which visually shows the effectiveness the method. The displayed sagittal slices are located midway between the location of the sparse samples. Figure 17 also highlights the resulting excellent CNR characteristic of the propagated static volume in the testing dataset. Animations of the resulting motion are provided as supplementary information and visually demonstrates the effectiveness of the method on the validating (section 3.1) and testing (section 3.2) datasets. We also highlight the resulting excellent CNR characteristic of a propagated static volume (figure 17) in the testing dataset. 


\section{Conclusion}

The major contribution from this work is the first demonstration that sparse sampling of free-breathing data may be used within a manifold learning paradigm to estimate fully sampled motion. To illustrate this it is demonstrated that the sparse motion model using a Bayesian framework with manifold (Procrustes) alignment can estimate equivalent motion vector fields compared to fully sampled 4D dynamic data, this allows a corresponding static volume to be articulated whilst preserving its high resolution / high contrast. Validation is performed on three 4D MRI datasets using 8 extracted slices from a fast $4 \mathrm{D}$ acquisitions ( $0.7 \mathrm{sec}$ per volume). The estimated deformation fields from sparse sampling are compared to the fully sampled equivalents over the entire field of view, resulting in an rms error of the order of $2 \mathrm{~mm}$. This work has also demonstrated the benefit of incorporating manifold alignment into a sparse motion model using Procrustes analysis versus no alignment. This alleviates the need for user definition of landmarks and provides a fully automated process. It was shown that on average the manifold alignment step reduces the mean root mean square error by $\approx 16 \%$. Exemplar $4 \mathrm{D}$ high contrast, high resolution articulated volunteer datasets using this methodology are also presented. In the clinical setting this approach facilitates greater freedom in the acquisition of free breathing respiratory motion sequences which may be used to inform motion modelling methods in a range of imaging modalities. In this current work we have utilized a high resolution static volume to articulate motion in this intra-modality setting. The manifold alignment framework however may be used to unify image contrast and motion estimates made from inter-modality settings. Such an approach can deliver superior CNR data than has hitherto been available. The resultant articulated high resolution / high contrast image has the advantage of allowing features from the static volume to be visualized in real time producing easier visualization and hence delineation of organs / tissues of interest. Furthermore the framework may alternatively aid in the optimization of acquisition sequences to further enhance temporal or spatial resolution inclusive of introducing new motion derived from the sparsely sampled data such as biopsy needle injections or other interventional procedures, which is an area of future work. The framework is also potentially generalizable, and so offers the ability to incorporate other external / internal surrogate signals to estimate motion which may provide additional flexibility when considering the need for a combination of varying imaging approaches during, for example, PET/MRI investigations, MR-guided treatments such as high intensity focused ultrasound or the guidance of radiotherapy treatments in hybrid MR-Linac devices.

\section{Appendix A. Derivation of Bayesian Model Fit}

Expanding upon the sparse motion model described in section 2.4, a Bayesian approach to fitting model parameters described in equation $14\left(\mathbf{h w}_{k} \mathbf{Q}=\mathbf{U}^{\prime *} \mathbf{r}\right)$, may be achieved. The Bayesian approach avoids over fitting, and permits the model to generalize to novel 
values and account for any wrong assumptions on $\mathbf{U}^{\prime}, \mathbf{h}$ and $\mathbf{Q}$. An expression (A.2) for the likelihood of observing the measured vector $\mathbf{r}_{k}$ can be obtained, assuming it is subject to uncorrelated Gaussian noise with a variance $\sigma_{N}^{2}$. Utilizing Bayes' rule and equation 6 then the probability of model parameters given the observed data is obtained (A.3).

$$
\begin{aligned}
& P\left(\mathbf{r} \mid \mathbf{r}_{\text {model }}\right) \propto e^{\frac{1}{2 \sigma_{N}^{2}}\left\|\mathbf{r}_{\text {model }}-\mathbf{r}\right\|^{2}} \\
& P(\mathbf{r} \mid \mathbf{w}) \propto \quad e^{\frac{1}{2 \sigma_{N}^{2}}\left\|\mathbf{U}^{\prime} \mathbf{w}-\mathbf{r}\right\|^{2}} \\
& P(\mathbf{w} \mid \mathbf{r}) \propto \quad e^{\frac{1}{2 \sigma_{N}^{2}}\left\|\mathbf{U}^{\prime} \mathbf{w}-\mathbf{r}\right\|^{2}} \cdot e^{\frac{1}{2}\|w\|^{2}}
\end{aligned}
$$

As the model parameters sought are those in the domain of the dense field we add an additional Procrustes alignment step (equation A.4) with $w^{\prime}=h w Q$.

$$
P\left(w^{\prime} \mid r\right) \propto e^{\frac{1}{2 \sigma_{N}^{2}}\left\|U^{\prime} h w Q-r\right\|^{2}} e^{\frac{1}{2}\|h w Q\|^{2}}
$$

If the sparse samples were already extracted from the domain of the dense field this would result in a unity transform, i.e $w^{\prime}=w$. Taking the logarithm of equation A.4 allows the definition of the cost function (equation A.7) to be minimized via equation A.7.

$$
\begin{aligned}
& E=-2 . \log P\left(w^{\prime} \mid r\right) \\
& E=\frac{1}{\sigma_{N}^{2}}\left\|U^{\prime} w-r\right\|^{2}+\left\|w^{\prime}\right\|^{2}+\text { const } \\
& E=\left\|U^{\prime} w^{\prime}-r\right\|^{2}+\eta \cdot\left\|w^{\prime}\right\|^{2}
\end{aligned}
$$

Expansion of equation A.7 results in:

$$
\begin{aligned}
& E=<U^{\prime} w^{\prime}, U^{\prime} w^{\prime}>-2<U w^{\prime}, r>+\|r\|^{2}+\eta \cdot\left\|w^{\prime}\right\|^{2} \\
& E=<w^{\prime}, U^{\prime T} U^{\prime} w^{\prime}>-2<w^{\prime}, U^{\prime T}, r>+\|r\|^{2}+\eta \cdot\left\|w^{\prime}\right\|^{2} \\
& 0=\nabla E=2 U^{\prime T} U^{\prime} w^{\prime}-2 U^{\prime T} r+2 \eta w^{\prime} \\
& \text { therefore: } \\
& U^{\prime T} U^{\prime} w^{\prime}+\eta w^{\prime}=U^{\prime T} w^{\prime}
\end{aligned}
$$

Singular value decomposition of $U^{\prime}=S A V^{T}$ with $A=\operatorname{diag}\left(a_{1}, a_{2}, . . a_{i}\right)$ gives $U^{\prime T} U^{\prime}=$ $S A^{2} S^{T}$, substituting into equation A.8 and multiplying by $V^{T}$ allows a solution for $w^{\prime}$ in a single step to be derived.

$$
\begin{aligned}
& A^{2} V^{T} w^{\prime}+\eta V^{T} w^{\prime}=A S^{T} r \\
& \operatorname{diag}\left(a_{i}^{2}+\eta\right) V^{T} w^{\prime}=A S^{T} r \\
& V^{T} w^{\prime}=\operatorname{diag}\left(\frac{a_{i}}{a_{i}^{2}+\eta}\right) S^{T} r \\
& w^{\prime}=V \operatorname{diag}\left(\frac{a_{i}}{a_{i}^{2}+\eta}\right) S^{T} r
\end{aligned}
$$

Therefore :

$$
w=h\left(V \operatorname{diag}\left(\frac{a_{i}}{a_{i}^{2}+\eta}\right) S^{T} r\right) Q
$$


The regularization factor $\eta=\sigma_{N}^{2} \geq 0$ acts as a trade off between prior probability of the solution and matching quality of the model fit, which in the presence of noise in $r$ or wrong assumptions of $U^{\prime}$ may result in heavily distorted solutions $v_{k}$. With appropriate choice of $\eta$, SVD of the reduced eigenvectors and Procrustes analysis to find values of $h$ and $Q$, then an optimal value of $w$ and hence $x$ may be found (equation A.11).

$$
x=U h\left(\operatorname{Vdiag}\left(\frac{a_{i}}{a_{i}^{2}+\eta}\right) S^{T} r\right) Q
$$




\section{References}

Advanced Normalization Tools ( ANTS ) (2014). Technical report, Universities of Pennsylvania, Virginia and Iowa.

Avants, B. B., Epstein, C. L., Grossman, M. and Gee, J. C. (2008). Symmetric Diffeomorphic Image Registrationn with Cross Correlation: Evaluating Automated Labelling of Elderly and Neurodegenerative Brain, Med Image Anal. 12(1): 26-41.

Avants, B. B. et al. (2008). Symmetric diffeomorphic image registration with cross correlation: evaluating automated labelling of elderly and neurodegenerative brain, Med. Image Anals. 12(1): 26-41.

Bai, W. et al. (2009). Regularized B-spline deformable registration for respiratory motion correction in PET images, Phys. Med. Biol. 54(9): 2719-2736.

Barnes, P., Baldock, C., Meikle, S. and Fulton, R. (2008). Benchmarking of a Motion Sensing System for Medical Imaging and Radiotherapy, Physics in Med. Biol. 53(20): 5845-5857.

Baumgartner, C. F. et al. (2014). Groupwise simultaneous manifold alignment for highresolution dynamic MR imaging of respiratory motion, Med. Image Anal. 18: 939-52.

Baumgartner, C., Kolbitsch, C., McClelland, J., Rueckert, D. and King, A. (2017). Autoadaptive motion modelling for MR-based respiratory motion estimation, Medical Image Analysis 51(35): 83-100.

Biederer, J., Eichinger, M. and J, D. (2017). Dynamic MRI of Respiratory Mechanics and Pulmonary Motion, Springer, Cham.

Blackall, J., Ahmad, S., Miquel, M., McClelland, J., Landau, D. and DJ, H. (2006). MRI-based measurements of respiratory motion variability and assessment of imaging stratergies for radiotherapy planning, Physics in Med. Biol. 51(17): 4147-4169.

Blanz, V. et al. (2002). Reconstructing the complete 3D shape of faces from partial information, Information Technology 44(6): 295.

Buerger, C., Schaeffter, T. and King, A. P. (2011). Hierarchical adaptive local affine registration for fast and robust respiratory motion estimation., Medical Image Analysis 15(4): 551-64.

Cai, J. et al. (2011). Four-dimensional magnetic resonance imaging (4D MRI) using image-based respiratory surrogate: A feasibility study, Med. Phys. 38(12): 6384-6394.

Dawood, M., Lang, N., Jiang, X. and Schafers, K. (2006). Lung motion correction on respiratory gated 3-D PET/CT images., IEEE Transactions on Medical Imaging 25(4): 476-85.

Dikaios, N. et al. (2014). MRI-based motion correction of thoracic PET: initial comparison of acquisition protocols and correction stratergies suitable for simultaneous PET/MRI systems, Eur. J Radiol. 22(2): 439-446.

Faranesh, A. Z. et al. (2013). Integration of cardiac and respiratory motion into MRI roadmaps fused with x-ray, Med. Phys. 40(3): 032302-8. 
Fayad, H., Odille, H., Schmidt, H., Wurslin, C., Kustner, T., Jacques, F. and Dimitris, V. (2015). The Use of a Generalized Reconstriction by Inversion of Coupled Systems (GRICS) Approach for Generic Respiratory Motion Correction in PET/MR Imaging, Physics in Med. Biol. 60(6): 2529-2546.

Fayad, H., Pan, T., Pradier, O. and Visvikis, D. (2012). Patient Specific Respiratory Motion Modeling Using a 3D Patients External Surface, Med Phys. 39(6): 3386-95.

Hollingsworth, K. et al. (2015). Reducing Acquisition Time in Clinical MRI by Data UNdersampling and Compressed Sensing Reconstruction, Physics in Med. Biol. 60: $297-322$.

Keall, P. J., Mageras, G. S., Balter, J. M., Emery, R. S., Forster, K. M., Jiang, S. B., Kapatoes, J. M., Low, D. a., Murphy, M. J., Murray, B. R., Ramsey, C. R., Van Herk, M. B., Vedam, S. S., Wong, J. W. and Yorke, E. (2006). The Management of Respiratory Motion in Radiation Oncology Report of AAPM Task Group 76, Med Phys 33(10): 3874-900.

King, A. P., Buerger, C., Tsoumpas, C., Marsden, P. K. and Schaeffter, T. (2012). Thoracic Respiratory Motion Estimation from MRI Using a Statistical Model and a 2-D Image Navigator, Med Image Anal. 16(1): 252-264.

King, A. P., Rhode, K. S., Razavi, R. S. and Schaeffter, T. R. (2009). An adaptive and predictive respiratory motion model for image-guided interventions: theory and first clinical application., IEEE Transactions on Medical Imaging 28(12): 2020-32.

Kustner, T. et al. (2016). MR Image Reconstruction Using a Combination of Compressed Sensing and Partial Fourier Acquisitions:ESPReSSO, IEEE Transaction on Medical Imaging. 35: 2447-2458.

Lagendijk, J. J. (2007). MR guidance in radiotherapy, Physics in Med. Biol. 59: R349. Liu, C. et al. (2011). Respiratory motion correction for quantitative PET/CT using all detected events with internal-externals motion correlation, Med. Phys. 38(5): 2715.

Maaten, L. J. P. V. D., Postma, E. O. and Herik, H. J. V. D. (2008). Dimensionality Reduction : A Comparative Review, Technical Report January, Tilburg University, Tilburg.

Mcclelland, J. R., Champion, B. A. S. and Hawkes, D. J. (2014). Combining Image Registration , Respiratory Motion Modelling, and Motion Compensated Image Reconstruction, MICCAI.

McClelland, J. R. et al. (2012). Respiratory motion models: A review, Med. Image Anals. 17(1): 19-42.

Polycarpou, I. et al. (2014). Impact of respiratory motion correction and spatial resolution on lesion detection in PET: a simulation study based on real MR dynamic data, Phys. Med. Biol. 59(3): 697-713.

Preiswerk, F., Arnold, P., Fasel, B. and Cattin, P. C. (2012). A Bayesian Framework for Estimating Respiratory Liver Motion from Sparse Measurements, Abdominal Imaging. Computational and Clinical Applications 7029: 207-214. 
Smith, R. et al. (2013a). Dense motion propogation from sparse samples for free breathing respiratory motion modelling, IEEE NSS/MIC conf. rec. pp. 1-5.

Smith, R. et al. (2013b). Toward a framework for high resolution parametric respiratory motion modelling, IEEE NSS/MIC conf. rec. pp. 1-4.

Tahavori, F., Alnowami, M. and Wells, K. (2014). Marker-less respiratory motion modelling using the microsoft kinect for windows', SPIE Medical Imaging Conf., Vol. 9036.

Tsoumpas, C. et al. (2011). Fast generation of 4D PET-MR data from real dynamic MR acquisitions, Phys. Med. Biol. 56(20): 6597-613.

von Siebenthal, M. et al. (2007). 4D MR imaging of respiratory organ motion and its variability, Physics in Med. Biol. 52(6): 1547-64.

Wang, C. and Mahadevan, S. (2008). Manifold Alignment Using Procrustes Analysis Manifold Alignment using Procrustes Analysis, Conf. MACH LEARN, pp. 1-8.

Wilms, M., Werner, R., Ehrhardt, J., Schmidt-Richberg, A., Blendowski, M. and Handels, H. (2013). Surrogate-based diffeomorphic motion estimation for radiation therapy: comparison of multivariate regression approaches, SPIE Medical Imaging Conf., Vol. 8669, pp. 866915-866918.

$\mathrm{Xu}, \mathrm{Q}$. et al. (2011). Respiratory motion blur identification and reduction in ungated thoracic PET imaging, Phys. Med. Biol. 56(14): 4481-4498. 